\title{
Multiloop soft theorem for gravitons and dilatons in the bosonic string
}

\author{
Paolo Di Vecchia, ${ }^{a, b}$ Raffaele Marotta ${ }^{c}$ and Matin Mojaza ${ }^{d}$ \\ ${ }^{a}$ The Niels Bohr Institute, University of Copenhagen, \\ Blegdamsvej 17, DK-2100 Copenhagen Ø, Denmark \\ ${ }^{b}$ Nordita, KTH Royal Institute of Technology and Stockholm University, \\ Roslagstullsbacken 23, SE-10691 Stockholm, Sweden \\ ${ }^{c}$ Istituto Nazionale di Fisica Nucleare, Sezione di Napoli, \\ Complesso Universitario di Monte S. Angelo ed. 6, via Cintia, 80126, Napoli, Italy \\ ${ }^{d}$ Max-Planck-Institut für Gravitationsphysik, Albert-Einstein-Institut, \\ Am Mühlenberg 1, 14476 Potsdam, Germany \\ E-mail: divecchi@nbi.ku.dk, raffaele.marotta@na.infn.it, \\ matin.mojaza@aei.mpg.de
}

ABSTRACT: We construct, in the closed bosonic string, the multiloop amplitude involving $N$ tachyons and one massless particle with $26-D$ compactified directions, and we show that at least for $D>4$, the soft behaviors of the graviton and dilaton satisfy the same soft theorems as at the tree level, up to one additional term at the subsubleading order, which can only contribute to the dilaton soft behavior and which we show is zero at least at one loop. This is possible, since the infrared divergences due to the non-vanishing tachyon and dilaton tadpoles do not depend on the number of external particles and are therefore the same both in the amplitude with the soft particle and in the amplitude without the soft particle. Therefore this leaves unchanged the soft operator acting on the amplitude without the soft particle. The additional infrared divergence appearing for $D \leq 4$ depend on the number of external legs and must be understood on their own.

Keywords: Bosonic Strings, Scattering Amplitudes

ARXIV EPRINT: 1808.04845 


\section{Contents}

1 Introduction 1

2 Multiloop amplitude in the bosonic string 4

2.1 The $h$-loop $N$-Reggeon vertex 4

$2.2 h$-loop amplitude with $N$ tachyons and one massless state 6

3 Soft behavior of a massless closed string at multiloops $\quad 8$

$\begin{array}{lll}3.1 & \text { All-loop graviton soft theorem } & 11\end{array}$

$\begin{array}{lll}3.2 & \text { All-loop dilaton soft theorem } & 11\end{array}$

4 Infrared divergences $\quad 13$

5 Conclusions 16

$\begin{array}{ll}\text { A On the gravitational coupling constant in string theory } & 17\end{array}$

$\begin{array}{ll}\text { B Schottky parametrization of Riemann surfaces } & 19\end{array}$

$\begin{array}{ll}\text { B.1 The Schottky group } & 19\end{array}$

B.2 The Abelian differentials and the period matrix 21

$\begin{array}{lll}\text { B.3 The prime form } & 22\end{array}$

$\begin{array}{ll}\text { C Scalar Green function on genus } h \text { Riemann surface } & 23\end{array}$

C.1 Choice of coordinates 24

C.2 Properties of the regularized Green function 25

$\begin{array}{ll}\text { D Derivation of the } N \text {-Reggeon vertex } & 28\end{array}$

$\begin{array}{lll}\text { E Calculation of } \boldsymbol{S}_{\mathbf{1}} & \mathbf{3 0}\end{array}$

E.1 Calculation of $I_{0} \quad 31$

E.2 Calculation of $I_{1}$

$\begin{array}{lll}\text { E.3 Calculation of } I_{2} & 37\end{array}$

$\begin{array}{lll}\text { F } & \text { Calculation of } S_{2}^{(1)} & 38\end{array}$

\section{Introduction}

Soft theorems for gravitons and other massless particles at the tree level have been intensively studied in the last few years both in field theory [1-34] and in string theory [35-44]. In particular, in string theory, it has been shown that, the leading, subleading and subsubleading behavior of a soft dilaton is universal, i.e. it is the same in any string theory, while, 
in the case of the graviton, the subsubleading behavior has, in general, string corrections that depend on the string theory under consideration $[45,46]$. In both cases those soft theorems are a direct consequence of gauge invariance [47-49] and of the structure of the three-point vertex containing a soft particle and two hard particles [50]. Gauge invariance fixes also the leading soft behavior of the Kalb-Ramond field [51].

At loop level it has been observed that the tree soft theorems are modified by infrared and ultraviolet divergences occurring in field theory [52-54], but, if one considers gravity theories that are ultraviolet complete and free from infrared divergences, by taking the number of space-time dimensions $D>4$, then one gets again a universal soft behavior for gravitons up to subleading level [55], and a factorizing soft behavior through subsubleading order, where universality is broken only by the two- and three-point coupling of the soft graviton to the other fields of the underlying theory [56].

In this paper we consider the $h$-loop amplitude of the closed bosonic string involving $N$ tachyons and one massless state and we show that we obtain the same soft theorems for gravitons, as at tree-level, as well as for the dilaton once its soft operator is rewritten in terms of the scaling properties of the amplitude, as long as we keep the non-compact number of the space-time dimensions, $D$, to be greater than four, and up to possibly an additional term at the subsubleading order, which, however, we have been able to show to be zero at one loop.

As in refs. $[55,56]$ we need $D>4$ in order to avoid infrared divergences that depend on the number of external legs [52]. On the other hand, working in a string theory, we have no ultraviolet divergences. We have, however, in the bosonic string infrared divergences due to the dilaton tadpole. These divergences are, however, not dependent on the number of the external states and therefore appear both in the multiloop amplitude with $N$ tachyons and one massless state and in the amplitude with only $N$ tachyons leaving the soft operator connecting them unaffected by these divergences.

The $h$-loop amplitude with $N$ tachyons and one massless state is obtained from the $h$-loop $(N+1)$-Reggeon vertex constructed in refs. [57-59], by means of the sewing procedure, starting from the tree diagram $(N+1)$-Reggeon vertex originally constructed by Lovelace [60] including the part with the ghost coordinates [57,61]. Multiloop amplitudes in the bosonic string were computed even before one realized that the extension of the Veneziano model was a string theory. The correct integration measure over the moduli was, however, only fixed in the eighties after the formulation of the BRST invariant action for the bosonic string theory [62-65]. It turns out that the $h$-loop amplitude with $N$ tachyons and one massless state, $\mathcal{M}_{N+1}^{(h)}$, except for one term, which is present only in the dilaton amplitude and that we will discuss later, has the same form in terms of the Green function, as in the case of the tree diagrams. This observation allows us to obtain the graviton soft theorem at the multiloop level as it was done in ref. [66] at the tree level. Because of this, the soft operator is the same at tree and loop level.

In the case of a soft dilaton the presence of the extra term gives a new type of contribution starting from the subleading order. We have been able to evaluate it at subleading 
order at the multiloop level, leading to the following soft theorem up to subleading order:

$$
M_{N ; \phi}^{(h)}\left(k_{i} ; q\right)=\frac{\kappa_{D}}{\sqrt{D-2}}\left[-\sum_{i=1}^{N} \frac{m^{2}}{k_{i} q} e^{q \partial_{k_{i}}}+2-\sum_{i=1}^{N} k_{i} \cdot \partial_{k_{i}}+h(D-2)\right] M_{N}^{(h)}+\mathcal{O}(q),
$$

where $\kappa_{D}$ is the gravitational constant in $D$ non-compact space-time dimensions (see appendix A for a definition), $m^{2}=-\frac{4}{\alpha^{\prime}}$ is the mass of the tachyons, and the operator $e^{q \partial_{k_{i}}}$ should be considered expanded up to $\mathcal{O}(q)$. Apart from the $h$-dependent term, this is the same operator as at tree-level.

At subsubleading order, we have evaluated the contribution from the extra term only at one loop, where it turns out to vanish as a consequence of momentum conservation. Hence the tree-level soft theorem is at this order unchanged at the one loop level, where the soft theorem reads

$$
M_{N ; \phi}^{(1)}\left(k_{i} ; q\right)=\frac{\kappa_{D}}{\sqrt{D-2}}\left[-\sum_{i=1}^{N} \frac{m^{2}}{k_{i} q} \mathrm{e}^{q \partial_{k_{i}}}+D-\sum_{i=1}^{N} \hat{D}_{i}+q_{\mu} \sum_{i=1}^{N} \hat{K}_{i}^{\mu}\right] M_{N}^{(1)}+\mathcal{O}\left(q^{2}\right),
$$

where we defined

$$
\hat{D}_{i}=k_{i} \cdot \frac{\partial}{\partial k_{i}}, \quad \hat{K}_{i}^{\mu}=\frac{1}{2} k_{i}^{\mu} \frac{\partial^{2}}{\partial k_{i \nu} \partial k_{i}^{\nu}}-k_{i}^{\rho} \frac{\partial^{2}}{\partial k_{i}^{\rho} \partial k_{i \mu}},
$$

the generators of the space-time dilatations and special conformal transformations.

The subleading soft operator differs from the tree-level one by the term explicitly dependent on the loop number $h$, which at first sight looks like an obstruction against a soft theorem for the full amplitude. It is, however, possible to recast the $h$-loop soft dilaton operator into a form that makes it the same at any number of loops. To this end, recall that the string amplitudes depend on three constants; the Regge slope $\alpha^{\prime}$, the string coupling constant $g_{s}$ and the gravitational coupling constant $\kappa_{D}$. Only two of them are fundamental and one can take $\kappa_{D}$ to be a function of the other two constants. In a compactified theory, there is additionally a dependence on the compactification parameters, such as the radii of the compact manifold, which we collectively denote by $R$, and $\kappa_{D}$ can be considered also a function of those parameters (see appendix A). By taking into account the explicit dependence of the scattering amplitudes on these fundamental constants, we can rewrite the dilatation operator entering the $h$-loop soft dilaton operator as a scaling operator in terms of the fundamental constants, as also originally done at tree-level in ref. [70]. In this way, one exactly gets rid of the $h$-dependence, and finds the following dilaton soft theorem, valid to subleading order, for the full all-loop amplitude, $\mathcal{M}_{N}=\sum_{h=0}^{\infty} M_{N}^{(h)}$, when the other particles are $N$ closed string tachyons:

$$
\mathcal{M}_{N ; \phi}\left(k_{i} ; q\right)=\frac{\kappa_{D}}{\sqrt{D-2}}\left[-\sum_{i=1}^{N} \frac{m^{2}}{k_{i} q} e^{q \partial_{k_{i}}}+\frac{D-2}{2} g_{s} \frac{\partial}{\partial g_{s}}-\sqrt{\alpha^{\prime}} \frac{\partial}{\partial \sqrt{\alpha^{\prime}}}-R \frac{\partial}{\partial R}\right] \mathcal{M}_{N}\left(k_{i}\right)+\mathcal{O}(q) .
$$

The paper is organized as follows. In section 2 we discuss the $h$-loop $N$-Reggeon vertex and from it we derive the $h$-loop amplitude involving $N$ tachyons and one massless state. 
In section 3 we derive the soft behavior of the amplitude. In section 4 we discuss the infrared divergences both those due to the tadpoles of the bosonic string amplitudes and the ones appearing when the theory is compactified to four dimensions. In section 5 we present our conclusions. In appendix $\mathrm{A}$ a derivation of the gravitational coupling constant in the compactified theory and its relation to $g_{s}$ and $\alpha^{\prime}$ is given. In appendix B we review the Schottky parametrization of Riemann surfaces, and in appendix $\mathrm{C}$ we discuss some properties of the multiloop Green function. Appendix D, E, and F, give calculational and technical details on expression given in the text.

\section{Multiloop amplitude in the bosonic string}

In this section we compute in the bosonic string, toroidally compactified on $\mathbb{R}^{1, D-1} \otimes \mathbb{T}^{26-D}$, the multiloop amplitude containing $N$ tachyons and one massless state from the multiloop $N$-Reggeon vertex. In particular, in the first subsection we write down the $N$-Reggeon vertex. It is derived from previous literature $[59,62]$ and some details of its derivation are put in appendix D. In the second subsection we use it to construct the multiloop amplitude involving $N$ tachyons and one massless state in the closed bosonic string.

\subsection{The $h$-loop $N$-Reggeon vertex}

We start by writing the $N$-Reggeon vertex for the closed bosonic string [59] (see appendix D for details of the derivation from the expression in ref. [59]):

$$
\begin{aligned}
& \mathcal{V}_{N}=C_{h}\left(N_{0}\right)^{N} \int d V_{N}\langle\Omega| \exp \left[\left.\frac{1}{2} \sum_{i=1}^{N} \sum_{n=0}^{\infty} \frac{\alpha_{n}^{(i)}}{n !} \alpha_{0}^{(i)} \frac{\partial^{n}}{\partial z^{n}} \log V_{i}^{\prime}(z)\right|_{z=0}\right] \\
& \times \exp \left[\left.\frac{1}{2} \sum_{i=1}^{N} \sum_{n=0}^{\infty} \frac{\bar{\alpha}_{n}^{(i)}}{n !} \alpha_{0}^{(i)} \frac{\partial^{n}}{\partial \bar{z}^{n}} \log \bar{V}_{i}^{\prime}(\bar{z})\right|_{\bar{z}=0}\right] \\
& \times \exp \left[\left.\frac{1}{2} \sum_{i \neq j} \sum_{n, m=0}^{\infty} \frac{\alpha_{n}^{(i)}}{n !} \partial_{z}^{n} \partial_{y}^{m} \log \frac{E\left(V_{i}(z), V_{j}(y)\right)}{\sqrt{V_{i}^{\prime}(0) V_{j}^{\prime}(0)}}\right|_{z=y=0} \frac{\alpha_{m}^{(j)}}{m !}\right] \\
& \times \exp \left[\left.\frac{1}{2} \sum_{i \neq j} \sum_{n, m=0}^{\infty} \frac{\bar{\alpha}_{n}^{(i)}}{n !} \partial_{\bar{z}}^{n} \partial_{\bar{y}}^{m} \log \frac{E\left(\bar{V}_{i}(\bar{z}), \bar{V}_{j}(\bar{y})\right)}{\sqrt{\bar{V}_{i}^{\prime}(0) \bar{V}_{j}^{\prime}(0)}}\right|_{z=y=0} \frac{\bar{\alpha}_{m}^{(j)}}{m !}\right] \\
& \times \exp \left[\left.\frac{1}{2} \sum_{i=1}^{N} \sum_{n, m=0}^{\infty} \frac{\alpha_{n}^{(i)}}{n !} \partial_{z}^{n} \partial_{y}^{m} \log \frac{E\left(V_{i}(z), V_{i}(y)\right)}{V_{i}(z)-V_{i}(y)}\right|_{z=y=0} \frac{\alpha_{m}^{(i)}}{m !}\right] \\
& \times \exp \left[\left.\frac{1}{2} \sum_{i=1}^{N} \sum_{n, m=0}^{\infty} \frac{\bar{\alpha}_{n}^{(i)}}{n !} \partial_{\bar{z}}^{n} \partial_{\bar{y}}^{m} \log \frac{E\left(\bar{V}_{i}(\bar{z}), \bar{V}_{i}(\bar{y})\right)}{\bar{V}_{i}(\bar{z})-\bar{V}_{i}(\bar{y})}\right|_{\bar{z}=\bar{y}=0} \frac{\bar{\alpha}_{m}^{(i)}}{m !}\right] \\
& \times \exp \left[\sum_{i, j=1}^{N} \sum_{n=0}^{\infty}\left(\frac{\alpha_{n}^{(i)}}{n !} \partial_{z}^{n}+\frac{\bar{\alpha}_{n}^{(i)}}{n !} \partial_{\bar{z}}^{n}\right) \operatorname{Re}\left(\int_{z_{0}}^{V_{i}(z)} \omega_{I}\right)(2 \pi \operatorname{Im} \tau)_{I J}^{-1}\right. \\
& \left.\times \sum_{m=0}^{\infty}\left(\frac{\alpha_{m}^{(j)}}{n !} \partial_{z}^{m}+\frac{\bar{\alpha}_{m}^{(i)}}{m !} \partial_{\bar{z}}^{m}\right) \operatorname{Re}\left(\int_{z_{0}}^{V_{j}(y)} \omega_{J}\right)\right],
\end{aligned}
$$


where

$$
\alpha_{n}=a_{n} \sqrt{n} \quad \text { if } n \neq 0, \quad \alpha_{0}=\bar{\alpha}_{0}=\frac{\sqrt{2 \alpha^{\prime}}}{2} p,
$$

with $p$ being at this level still an operator. The functions $V_{i}(z)$, satisfying the condition $V_{i}(0)=z_{i}$, parametrize the coordinates around the various punctures (see appendix C.1 for details). It can be seen that, if the external states are on-shell physical states, the dependence on the $V_{i}(z)$ drops out. In the following we keep, however, the variables $V_{i}(z)$ in order to define a proper Green function. The quantity $E(z, y)$ is the prime form, $\omega_{I}$ are the abelian differentials for $I=1 \ldots h$ with $h$ being the genus of the Riemann surface, and $\tau$ is the period matrix. Repeated capital indices $I, J, \ldots$ are assumed to be summed over from $1, \ldots, h$. All these quantities are defined and discussed in appendix B. The constants $C_{h}$ and $N_{0}$ provide the correct normalization of the amplitude, and are equal to

$$
C_{h}=C_{0} N_{0}^{2 h}\left(\frac{\alpha^{\prime}}{8 \pi}\right)^{h} \frac{1}{\left(2 \pi \alpha^{\prime}\right)^{\frac{D h}{2}}}=\left(\frac{8 \pi}{\alpha^{\prime}}\right)^{1-h}\left(\frac{\kappa_{D}}{2 \pi}\right)^{2(h-1)} \frac{1}{\left(2 \pi \alpha^{\prime}\right)^{\frac{h D}{2}}} ; \quad N_{0}=\frac{\kappa_{D}}{2 \pi} .
$$

These expressions follow from the sewing procedure, which allows to obtain $h$-loop amplitudes from tree-level ones by sewing together $2 h$ external legs with the propagator

$$
\frac{\alpha^{\prime}}{8 \pi} \int_{|z| \leq 1} \frac{d^{2} z}{|z|^{2}} z^{L_{0}-1} \bar{z}^{\bar{L}_{0}-1}
$$

to produce $h$ handles (or loops). The first factor, $C_{0}$, on the left hand side of eq. (2.3) takes in account the normalization of the tree-level amplitude, the second factor, $N_{0}^{2 h}$, is the normalization of the sewed $2 h$ legs, the third factor, $\left(\alpha^{\prime} / 8 \pi\right)^{h}$, comes from the normalization of the propagator in eq. (2.4), while the last factor, $\left(2 \pi \alpha^{\prime}\right)^{-D h / 2}$, arises from the integration over the momenta circulating in the loops.

The measure of the moduli, in the Schottky parametrization of the Riemann surface, including the compactification factor, is equal to $[62-65]^{1}$

$$
\begin{aligned}
d V_{N}= & \prod_{i=1}^{N}\left(\frac{d^{2} z_{i}}{\left|V_{i}^{\prime}(0)\right|^{2}}\right) \frac{1}{d V_{a b c}} \prod_{I=1}^{h}\left[\frac{d^{2} \kappa_{I} d^{2} \xi_{I} d^{2} \eta_{I}}{\left|\kappa_{I}\right|^{4}\left|\xi_{I}-\eta_{I}\right|^{4}}\left|1-\kappa_{I}\right|^{4}\right](\operatorname{det} 2 \pi \operatorname{Im} \tau)^{-\frac{D}{2}} \\
& \times \prod_{\alpha}^{\prime}\left[\prod_{n=1}^{\infty}\left|\frac{1}{1-\kappa_{\alpha}^{n}}\right|^{52} \prod_{n=2}^{\infty}\left|1-\kappa_{\alpha}^{n}\right|^{4}\right][F(\tau, \bar{\tau})]^{26-D}
\end{aligned}
$$

Here $d V_{a b c}$ is the volume element of the $\mathrm{SL}(2, \mathbb{C})$ Möbius group, $\xi_{I}$ and $\eta_{I}$ are the attractive and repulsive fixed points and $\kappa_{I}$ is the multiplier of the $h$ generators of the Schottky group. $^{2} \kappa_{\alpha}$ is the multiplier of a primary class and $\prod_{\alpha}^{\prime}$ is a product over primary classes (see appendix E of ref. [59] for details). The factor $(\operatorname{det}(2 \pi \operatorname{Im} \tau))^{-\frac{D}{2}}$ in the measure comes from the integral over the momenta along the non-compact directions circulating in the loops. For the momenta along the compact dimensions one must replace the integral over

\footnotetext{
${ }^{1}$ In this work we use the convention $d^{2} z_{i}=2 \operatorname{dRe}\left(z_{i}\right) \operatorname{dIm}\left(z_{i}\right)$.

${ }^{2}$ We hope that the multipliers $\kappa_{I}$ are not confused with the gravitational constant $\kappa_{D}$.
} 
the momenta with a sum over the Kaluza-Klein modes $\mathbf{n}$ and the winding numbers $\mathbf{m}$. Therefore, for each compact dimension, the factor $(\operatorname{det}(2 \pi \operatorname{Im} \tau))^{-\frac{1}{2}}$ is replaced by [67]:

$$
F(\tau, \bar{\tau})=\sum_{(\mathbf{m}, \mathbf{n}) \in \mathbb{Z}^{2 h}} e^{i \pi\left(\mathbf{p}_{\mathbf{R}} \tau \mathbf{p}_{\mathbf{R}}-\mathbf{p}_{\mathbf{L}} \bar{\tau} \mathbf{p}_{\mathbf{L}}\right)},
$$

where $\mathbf{p}_{\mathbf{R} ; \mathbf{L}}=\frac{1}{\sqrt{2}}\left(\frac{\sqrt{\alpha^{\prime}}}{R} \mathbf{n} \pm \frac{R}{\sqrt{\alpha^{\prime}}} \mathbf{m}\right)$. Here $R$ denotes collectively the compactification radii.

Finally, the vacuum state is defined by:

$$
\langle\Omega| \equiv \prod_{i=1}^{N}\left[{ }_{i}\left\langle x=0 ; 0_{a}, 0_{\bar{a}}\right|\right](2 \pi)^{D} \delta^{(D)}\left(\sum_{i=1}^{n} p_{i}\right) .
$$

It should in principle also depend on the winding numbers and Kaluza-Klein modes of the compact dimension, but they are now irrelevant, and thus suppressed, since, in our case, the external states have momenta and oscillators only along non-compact directions.

We conclude this section by observing that in a compact space, the $h$-loop $N$-Reggeon Vertex depends also on the annihilation operators associated to the $26-D$ compact directions and on the left and right compact momentum operators. These should be included in eq. (2.1) but we have neglected them because they are irrelevant in our calculation. The physical states, tachyons and massless states of the closed string, considered in this paper are vacuum states along the compact directions with zero winding number and KaluzaKlein momenta. Therefore there are no contributions to the amplitude coming from these compact degrees of freedom. The only dependence from the compact directions is the one due to right and left discrete momenta circulating in the loop which has been properly taken in account in eq. (2.6).

\section{$2.2 \quad h$-loop amplitude with $N$ tachyons and one massless state}

We now specialize the vertex in eq. (2.1) for tachyons and massless states. We make the transition $N \rightarrow N+1$, since we will in the end specify $N$ states to be tachyons and one to be a massless state, but for now the $N+1$ states can be any of the two. For these states the vertex in eq. (2.1) reduces to

$$
\begin{aligned}
\mathcal{V}_{N+1}= & C_{h}\left(N_{0}\right)^{N+1} \int d V_{N+1}\langle\Omega| \exp \left[\frac{1}{2} \sum_{i \neq j=1}^{N+1}\left(\frac{\sqrt{2 \alpha^{\prime}}}{2} p_{i}+V_{i}^{\prime}(0) a_{1}^{(i)} \partial_{z_{i}}+\bar{V}_{i}^{\prime}(0) \bar{a}_{1}^{(i)} \partial_{\bar{z}_{i}}\right)\right. \\
& \left.\times\left(\frac{\sqrt{2 \alpha^{\prime}}}{2} p_{j}+V_{j}^{\prime}(0) a_{1}^{(i)} \partial_{z_{j}}+\bar{V}_{j}^{\prime}(0) \bar{a}_{1}^{(j)} \partial_{\bar{z}_{j}}\right) \log \frac{\left|E\left(z_{i}, z_{j}\right)\right|^{2}}{\left|V_{i}^{\prime}(0) V_{j}^{\prime}(0)\right|}\right] \\
& \times \exp \left[\sum_{i, j=1}^{N+1}\left(\frac{\sqrt{2 \alpha^{\prime}}}{2} p_{i}+V_{i}^{\prime}(0) a_{1}^{(i)} \partial_{z_{i}}+\bar{V}_{i}^{\prime}(0) \bar{a}_{1}^{(i)} \partial_{\bar{z}_{i}}\right) \operatorname{Re}\left(\int_{z_{0}}^{z_{i}} \omega_{I}\right)(2 \pi \operatorname{Im} \tau)_{I J}^{-1}\right. \\
& \left.\times\left(\frac{\sqrt{2 \alpha^{\prime}}}{2} p_{j}+V_{j}^{\prime}(0) a_{1}^{(j)} \partial_{z_{j}}+\bar{V}_{j}^{\prime}(0) \bar{a}_{1}^{(j)} \partial_{\bar{z}_{j}}\right) \operatorname{Re}\left(\int_{z_{0}}^{z_{j}} \omega_{J}\right)\right] .
\end{aligned}
$$

The exponentials in the first, second, fifth and sixth lines of eq. (2.1) do not contribute for the tachyon states. They also do not contribute for the massless states because in this case 
one obtains terms proportional to $(q \epsilon)$ that are zero for physical massless states ( $q$ is the momentum of the massless state and $\epsilon$ its polarization).

Separating in the last two lines terms with $i \neq j$ from those with $i=j$ and eliminating the dependence on $z_{0}$ as showed in appendix $\mathrm{D}$, we can write the previous equation as follows:

$$
\begin{aligned}
\mathcal{V}_{N+1}=C_{h}\left(N_{0}\right)^{N+1} \int d V_{N+1} & \langle\Omega| \exp \left[\frac{1}{2} \sum_{i \neq j=1}^{N+1}\left(\frac{\sqrt{2 \alpha^{\prime}}}{2} p_{i}+V_{i}^{\prime}(0) a_{1}^{(i)} \partial_{z_{i}}+\bar{V}_{i}^{\prime}(0) \bar{a}_{1}^{(i)} \partial_{\bar{z}_{i}}\right)\right. \\
\times & \left.\left(\frac{\sqrt{2 \alpha^{\prime}}}{2} p_{j}+V_{j}^{\prime}(0) a_{1}^{(j)} \partial_{z_{j}}+\bar{V}_{j}^{\prime}(0) \bar{a}_{1}^{(j)} \partial_{\bar{z}_{j}}\right) \mathcal{G}_{h}\left(z_{i}, z_{j}\right)\right] \\
\times & \exp \left[\frac{1}{2} \sum_{i=1}^{N+1}\left|V_{i}^{\prime}(0)\right|^{2} \omega_{I}\left(z_{i}\right)(2 \pi \operatorname{Im} \tau)_{I J}^{-1} \bar{\omega}\left(\bar{z}_{J}\right) \alpha_{1}^{(i)} \bar{\alpha}_{1}^{(i)}\right],
\end{aligned}
$$

where

$$
\mathcal{G}_{h}\left(z_{i}, z_{j}\right)=\log \frac{\left|E\left(z_{i}, z_{j}\right)\right|^{2}}{\left|V_{i}^{\prime}(0) V_{j}^{\prime}(0)\right|}+\operatorname{Re}\left(\int_{z_{j}}^{z_{i}} \omega_{I}\right)(2 \pi \operatorname{Im} \tau)_{I J}^{-1} \operatorname{Re}\left(\int_{z_{i}}^{z_{j}} \omega_{J}\right) .
$$

When evaluated on on-shell external states the final result for the amplitude will, as a consequence of momentum conservation, not depend on the factor $\left|V_{i}(0) V_{j}^{\prime}(0)\right|$ included in $\mathcal{G}_{h}$. We have, however, included them in the definition of $\mathcal{G}_{h}$, since this allows us to identify it with the regularized Green function of ref. [68]. As shown in appendix C.1, by choosing conformal coordinates with metric $d s^{2}=\rho(z, \bar{z}) d z d \bar{z}$ to parametrize the Riemann surface around the punctures $z_{i}$, the functions $\left|V_{i}^{\prime}(0)\right|^{2}$ can be set equal to

$$
\left|V_{i}^{\prime}(0)\right|^{2}=\frac{1}{\rho\left(z_{i}, \bar{z}_{i}\right)}
$$

With this choice, the function $\mathcal{G}_{h}$ is exactly equal to minus the regularized Green function $G_{r}$ discussed in appendix C, from where it follows that $\mathcal{G}_{h}$ then satisfies

$$
\begin{aligned}
\partial_{z} \partial_{\bar{z}} \mathcal{G}_{h}(z, w) & =\pi \delta^{(2)}(z-w)-\frac{1}{2} \omega_{I}(z)(2 \pi \operatorname{Im} \tau)_{I J}^{-1} \bar{\omega}_{J}(\bar{z})+\frac{1}{2} \partial_{z} \partial_{\bar{z}} \log \rho(z, \bar{z}), \\
\int \mathrm{d}^{2} z \partial_{z} \partial_{\bar{z}} \mathcal{G}_{h}(z, w) & =0 .
\end{aligned}
$$

In the rest of this paper we shall assume the choice of coordinates in eq. (2.11).

The previous vertex is valid for any number of tachyons and massless states. In the following we restrict ourselves to the case of $N$ tachyons and one massless state. This means that we have to saturate it with the states given by ${ }^{3}$

$$
\prod_{i=1}^{N}\left[\left|0, k_{i}\right\rangle\right] a_{1 \mu}^{\dagger} \bar{a}_{1 \nu}^{\dagger}|0, q\rangle
$$

where the $N$ tachyons have momenta $k_{i}$ and the massless state momentum $q$.

\footnotetext{
${ }^{3}$ We are again suppressing the vacuum structure along the compact directions, but as discussed at the end of section 2.1, for our purposes they are irrelevant.
} 
After careful contractions and some rewriting we get the following expression for the $h$-loop amplitude for $h \geq 1$ :

$$
\begin{aligned}
M_{N ; 1}^{(h)}= & C_{h}\left(N_{0}\right)^{N+1} \int d V_{N} \prod_{i<j=1}^{N} \mathrm{e}^{\frac{\alpha^{\prime}}{2} k_{i} k_{j} \mathcal{G}_{h}\left(z_{i}, z_{j}\right)} \epsilon_{q}^{\mu} \bar{\epsilon}_{q}^{\nu} \int d^{2} z \prod_{\ell=1}^{N} \mathrm{e}^{\frac{\alpha^{\prime}}{2} k_{\ell} q \mathcal{G}_{h}\left(z, z_{\ell}\right)} \\
& \times\left[\frac{\alpha^{\prime}}{2} \sum_{i, j=1}^{N} k_{i \mu} k_{j \nu} \partial_{z} \mathcal{G}_{h}\left(z, z_{i}\right) \partial_{\bar{z}} \mathcal{G}_{h}\left(z, z_{j}\right)+\frac{1}{2} \eta_{\mu \nu} \omega_{I}(z)(2 \pi \operatorname{Im} \tau)_{I J}^{-1} \bar{\omega}_{J}(z)\right] .
\end{aligned}
$$

In comparison, the tree-amplitude for the scattering of a massless particle and $N$ tachyons in the bosonic string is given by

$$
\begin{aligned}
M_{N ; 1}^{(0)}= & C_{0}\left(N_{0}\right)^{N+1} \int \frac{\prod_{i=1}^{N} d^{2} z_{i}}{d V_{a b c}} \prod_{i<j=1}^{N} \mathrm{e}^{\frac{\alpha^{\prime}}{2} k_{i} k_{j} \mathcal{G}_{0}\left(z_{i}, z_{j}\right)} \epsilon_{q}^{\mu} \bar{\epsilon}_{q}^{\nu} \int d^{2} z \prod_{\ell=1}^{N} \mathrm{e}^{\frac{\alpha^{\prime}}{2} k_{i} q \mathcal{G}_{0}\left(z, z_{\ell}\right)} \\
& \times \frac{\alpha^{\prime}}{2} \sum_{i, j=1}^{N} k_{i \mu} k_{j \nu} \partial_{z} \mathcal{G}_{0}\left(z, z_{i}\right) \partial_{\bar{z}} \mathcal{G}_{0}\left(z, z_{j}\right)
\end{aligned}
$$

where $\mathcal{G}_{0}(z, w)=\log |z-w|^{2}$. Except for the second term in the square bracket in eq. (2.15), and for the integration measure and the integration region, the two expressions in eqs. (2.16) and (2.15) have the same form in terms of their Green function. Notice that the extra term contributes only when the massless state is a dilaton.

\section{Soft behavior of a massless closed string at multiloops}

Starting from eq. (2.15), we would like to study its soft behavior when the momentum carried by the massless state is much lower than the momenta of the tachyons. Let us first notice that the $h$-loop amplitude can be separated into a part describing just the tachyon scattering convoluted with the contributions to the scattering of the massless state i.e.

$$
\begin{aligned}
M_{N ; 1}^{(h)}= & M_{N}^{(h)} * S\left(q, k_{i} ; z_{i}\right), \\
M_{N}^{(h)}= & C_{h} N_{0}^{N} \int d V_{N} \prod_{i<j}^{N} e^{\frac{\alpha^{\prime}}{2} k_{i} k_{j} \mathcal{G}_{h}\left(z_{i}, z_{j}\right)}, \\
S\left(q, k_{i} ; z_{i}\right)= & N_{0} \int \mathrm{d}^{2} z \prod_{i=1}^{N} e^{\frac{\alpha^{\prime}}{2} k_{i} q \mathcal{G}_{h}\left(z_{i}, z\right)} \\
& \times \int \mathrm{d}^{2} \theta \exp \left\{\sum_{i=1}^{N} \sqrt{\frac{\alpha^{\prime}}{2}} k_{i}\left(\theta \epsilon \partial_{z}+\bar{\theta} \bar{\epsilon} \partial_{\bar{z}}\right) \mathcal{G}_{h}\left(z_{i}, z\right)\right\} \\
& \times \exp \left\{\frac{1}{2} \theta \epsilon \cdot \bar{\theta} \bar{\epsilon} \omega_{I}(z)(2 \pi \operatorname{Im} \tau)_{I J}^{-1} \bar{\omega}_{J}(\bar{z})\right\},
\end{aligned}
$$


where $*$ denotes a convolution of the integrals. On its own, $M_{N}^{(h)}$ is exactly the $h$-loop $N$-tachyon amplitude. The Grassmanian integral in $S$ is easy to perform, yielding

$$
\begin{aligned}
S\left(q, k_{i} ; z_{i}\right)=N_{0} \int \mathrm{d}^{2} z \prod_{i=1}^{N} e^{\frac{\alpha^{\prime}}{2} k_{i} q \mathcal{G}_{h}\left(z_{i}, z\right)} & {\left[\frac{\alpha^{\prime}}{2} \sum_{i, j=1}^{N}\left(k_{i} \epsilon\right)\left(k_{j} \bar{\epsilon}\right) \partial_{z} \mathcal{G}_{h}\left(z_{i}, z\right) \partial_{\bar{z}} \mathcal{G}_{h}\left(z_{j}, z\right)\right.} \\
& \left.+\frac{1}{2}(\epsilon \cdot \bar{\epsilon}) \omega_{I}(z)(2 \pi \operatorname{Im} \tau)_{I J}^{-1} \bar{\omega}_{J}(\bar{z})\right]
\end{aligned}
$$

We would like to compute $S$ through order $q$ in the soft momentum limit. The second term above vanishes for the graviton and the Kalb-Ramond antisymmetric field, but contributes in the case of the dilaton (because the polarization tensor is traced). We separate the two terms accordingly into $\left(N_{0} S_{1}\right)$ and $\left(N_{0} S_{2}\right)$, and expand them (partly) in $q$ as follows

$$
\begin{aligned}
S_{1}= & \int \mathrm{d}^{2} z\left[1+\sum_{j \neq i}^{N} \frac{\alpha^{\prime}}{2} k_{j} q \mathcal{G}_{h}\left(z_{i}, z\right)+\frac{1}{2}\left(\frac{\alpha^{\prime}}{2}\right)^{2} \sum_{j, l \neq i}^{N}\left(k_{j} q\right)\left(k_{l} q\right) \mathcal{G}_{h}\left(z_{j}, z\right) \mathcal{G}_{h}\left(z_{l}, z\right)\right] \\
& \times \frac{\alpha^{\prime}}{2} \sum_{i, j=1}^{N}\left(k_{i} \epsilon\right)\left(k_{j} \bar{\epsilon}\right) \partial_{z} \mathcal{G}_{h}\left(z_{i}, z\right) \partial_{\bar{z}} \mathcal{G}_{h}\left(z_{j}, z\right) e^{\frac{\alpha^{\prime}}{2} k_{i} q \mathcal{G}_{h}\left(z_{i}, z\right)}+\mathcal{O}\left(q^{2}\right) \\
S_{2}= & \int \mathrm{d}^{2} z\left[1+\sum_{i=1}^{N} \frac{\alpha^{\prime}}{2} k_{i} q \mathcal{G}_{h}\left(z_{i}, z\right)\right] \frac{\epsilon \cdot \bar{\epsilon}}{2} \omega_{I}(z)(2 \pi \operatorname{Im} \tau)_{I J}^{-1} \bar{\omega}_{J}(\bar{z})+\mathcal{O}\left(q^{2}\right) .
\end{aligned}
$$

Notice that the integrand of $S_{1}$ is expanded through order $q^{2}$, since the integration can bring down one order of $q$. We will only keep terms through order $q$ after integration.

Let us first notice that the first integral in $S_{2}$ immediately follows from the Riemann Bilinear Identity; since the abelian forms are closed holomorphic forms we have

$$
\int d^{2} z \omega_{I}(z) \bar{\omega}_{J}(\bar{z})=-i \sum_{\sigma=1}^{h}\left[\oint_{a_{\sigma}} \omega_{I} \oint_{b_{\sigma}} \bar{\omega}_{J}-\oint_{b_{\sigma}} \omega_{I} \oint_{a_{\sigma}} \bar{\omega}_{J}\right]=4 \pi\left(2 \pi \operatorname{Im} \tau_{I J}\right),
$$

where the definition of the abelian cycles, outlined in appendix B, was used. It follows that

$$
S_{2}=(\epsilon \cdot \bar{\epsilon})\left[2 \pi h+S_{2}^{(1)}+\mathcal{O}\left(q^{2}\right)\right],
$$

where $S_{2}^{(1)}$ denotes the contribution at order $q$, given by the integral

$$
S_{2}^{(1)}=\sum_{i=1}^{N} \frac{\alpha^{\prime}}{4} k_{i} q \int \mathrm{d}^{2} z \mathcal{G}_{h}\left(z_{i}, z\right) \omega_{I}(z)(2 \pi \operatorname{Im} \tau)_{I J}^{-1} \bar{\omega}_{J}(\bar{z}) .
$$

This quantity is discussed in appendix F. At one loop it turns out that the integration gives an expression independent of $z_{i}$, and hence the total expression vanishes as a consequence of momentum conservation, i.e. $\sum_{i=1}^{N} k_{i} \cdot q=-q^{2}=0$. At the multiloop level, we have not been able to evaluate this integral. We notice for future studies that if the one loop result holds at multiloops, it is not necessary to calculate the integral explicitly, but only to show that the integral is independent of $z_{i}$. 
To compute $S_{1}$ let us first remark that we can restrict to the case where the soft state is symmetrically polarized, since the amplitude of one Kalb-Ramond state and $N$ closed tachyons is anyways zero because of world-sheet parity conservation. ${ }^{4}$ In the case where the soft state is symmetrically polarized, it remarkably turns out that $S_{1}$ is computable through order $q$ by using only the identities in eq. (2.12) and eq. (2.13), i.e. independent of the explicit form of $\mathcal{G}_{h}$. We leave the details of this important result to the appendix $\mathrm{E}$ and here quote the final expression:

$$
\begin{aligned}
S_{1}=2 \pi \varepsilon_{q \mu \nu}^{S} \sum_{i=1}^{N} \frac{k_{i}^{\mu} k_{i}^{\nu}}{k_{i} q}+2 \pi \varepsilon_{q \mu \nu}^{S} \frac{\alpha^{\prime}}{2} \sum_{i \neq j}\left[\frac{k_{i}^{\mu} k_{i}^{\nu}}{k_{i} q}\left(k_{j} q\right) \mathcal{G}_{h}\left(z_{j}, z_{i}\right)-k_{i}^{\mu} k_{j}^{\nu} \mathcal{G}_{h}\left(z_{j}, z_{i}\right)\right] \\
+2 \pi \varepsilon_{q \mu \nu}^{S} \frac{1}{2}\left(\frac{\alpha^{\prime}}{2}\right)^{2} \sum_{i \neq j, l}\left[k_{i}^{\mu} k_{i}^{\nu} \frac{\left(k_{j} q\right)\left(k_{l} q\right)}{q k_{i}}+k_{j}^{\mu} k_{l}^{\nu}\left(k_{i} q\right)\right. \\
\left.-k_{i}^{\mu} k_{l}^{\nu}\left(k_{j} q\right)-k_{i}^{\mu} k_{j}^{\nu}\left(k_{l} q\right)\right] \mathcal{G}_{h}\left(z_{i}, z_{l}\right) \mathcal{G}_{h}\left(z_{i}, z_{j}\right)+\mathcal{O}\left(q^{2}\right) .
\end{aligned}
$$

Noticeably, this result is formally equal to the tree-level result found in eq. (2.5) of ref. [66], upon inserting the corresponding tree-level Green function. It follows that it is reproduced by the same soft theorem as valid at tree-level, which we can now easily check: the first term is just the Weinberg soft theorem, when multiplied with $N_{0}$, which immediately factorizes out of $M_{n}$, since it is independent on the Koba-Nielsen variables. The subleading terms should be reproduced by the following operation:

$$
\begin{aligned}
-i \kappa_{D} \varepsilon_{q \mu \nu}^{S} \sum_{i=1}^{N} \frac{k_{i}^{\mu} q_{\rho} L_{i}^{\nu \rho}}{k_{i} q} M_{N}^{(h)} & =\kappa_{D} \varepsilon_{q \mu \nu}^{S} \sum_{i=1}^{N} \frac{k_{i}^{\mu} q_{\rho}}{k_{i} q}\left(k_{i}^{\nu} \partial_{k_{i}}^{\rho}-k_{i}^{\rho} \partial_{k_{i}}^{\nu}\right) M_{N}^{(h)} \\
& =\kappa_{D} M_{N}^{(h)} * \varepsilon_{q \mu \nu}^{S} \sum_{i \neq j}^{N} \frac{k_{i}^{\mu} q_{\rho}}{k_{i} q}\left[k_{i}^{\nu} k_{j}^{\rho}-k_{i}^{\rho} k_{j}^{\nu}\right] \mathcal{G}_{h}\left(z_{i}, z_{j}\right) \\
& =\kappa_{D} M_{N}^{(h)} * \varepsilon_{q \mu \nu}^{S} \frac{\alpha^{\prime}}{2} \sum_{i \neq j}^{N}\left[\frac{k_{i}^{\mu} k_{i}^{\nu}}{k_{i} q}\left(k_{j} q\right)-k_{i}^{\mu} k_{j}^{\nu}\right] \mathcal{G}_{h}\left(z_{i}, z_{j}\right),
\end{aligned}
$$

which is exactly equal to the subleading soft term in eq. (3.8) when multiplied with $N_{0}$. Finally, the subsubleading tree-level soft operator reads [66]:

$$
\begin{aligned}
& -\kappa_{D} \varepsilon_{q \mu \nu}^{S} \sum_{i=1}^{N} \frac{q_{\rho} q_{\sigma}}{2 k_{i} q}: L_{i}^{\mu \rho} L_{i}^{\nu \sigma}: M_{N}^{(h)} \\
& =\kappa_{D} \varepsilon_{q \mu \nu}^{S} \sum_{i=1}^{N} \frac{q_{\rho} q_{\sigma}}{2 k_{i} q}\left(k_{i}^{\mu} k_{i}^{\nu} \partial_{k_{i}}^{\rho} \partial_{k_{i}}^{\sigma}+k_{i}^{\rho} k_{i}^{\sigma} \partial_{k_{i}}^{\mu} \partial_{k_{i}}^{\nu}-k_{i}^{\mu} k_{i}^{\sigma} \partial_{k_{i}}^{\nu} \partial_{k_{i}}^{\rho}-k_{i}^{\nu} k_{i}^{\rho} \partial_{k_{i}}^{\mu} \partial_{k_{i}}^{\sigma}\right) M_{N}^{(h)}
\end{aligned}
$$

\footnotetext{
${ }^{4}$ More precisely, world-sheet parity $\Omega$, which is a symmetry of the closed bosonic string, leaves invariant the vertex operators of the tachyon, dilaton and graviton, while changing sign of the Kalb-Ramond vertex operator.
} 


$$
\begin{aligned}
=M_{N}^{(h)} * \frac{\kappa_{D}}{2}\left(\frac{\alpha^{\prime}}{2}\right)^{2} \varepsilon_{q \mu \nu}^{S} \sum_{i=1} \sum_{j, l \neq i} & \left\{k_{i}^{\mu} k_{i}^{\nu} \frac{\left(k_{j} q\right)\left(k_{l} q\right)}{q k_{i}}+k_{j}^{\mu} k_{l}^{\nu}\left(k_{i} q\right)\right. \\
& \left.-k_{i}^{\mu} k_{l}^{\nu}\left(k_{j} q\right)-k_{i}^{\mu} k_{j}^{\nu}\left(k_{l} q\right)\right\} \mathcal{G}_{h}\left(z_{i}, z_{l}\right) \mathcal{G}_{h}\left(z_{i}, z_{j}\right),
\end{aligned}
$$

which is exactly equal to the subsubleading term in eq. (3.8) when multiplied with $N_{0}$. The term containing two $\mathrm{L}$ in the subsubleading soft operator above is normal ordered, hence the : : notation, meaning that the operator $L$ on the left acts on everything on its right except on the other $L$. The normal ordering is irrelevant for the graviton, but is important for getting the right behavior of the dilaton.

To summarize, we have found that the $h$-loop soft behavior of the graviton and dilaton when scattering with $N$ tachyons in the bosonic string can be written as:

$$
\begin{aligned}
M_{N ; 1}^{(h)}\left(k_{i} ; q\right)= & \kappa_{D} \varepsilon_{q \mu \nu}^{S} \sum_{i=1}^{N}\left[\frac{k_{i}^{\mu} k_{i}^{\nu}}{k_{i} q}-i \frac{k_{i}^{\mu} q_{\rho}}{k_{i} q} L_{i}^{\nu \rho}-\frac{q_{\rho} q_{\sigma}}{2 k_{i} q}: L_{i}^{\mu \rho} L_{i}^{\nu \sigma}:+h \eta^{\mu \nu}\right] M_{N}^{(h)} \\
& +(\epsilon \cdot \bar{\epsilon}) \frac{\kappa_{D}}{2 \pi}\left(M_{N}^{(h)} * S_{2}^{(1)}\right)+\mathcal{O}\left(q^{2}\right)
\end{aligned}
$$

The last term is zero at least at tree-level and at one loop.

\subsection{All-loop graviton soft theorem}

The full amplitude of one graviton and $N$ closed tachyons is

$$
\mathcal{M}_{N ; g}\left(k_{i} ; q\right)=\left.\sum_{h=0}^{\infty} M_{N ; 1}^{(h)}\left(k_{i} ; q\right)\right|_{\varepsilon_{q \mu \nu}^{S}=\varepsilon_{\mu \nu}^{g}}
$$

Since the polarization tensor of the graviton is traceless, $\varepsilon_{\mu \nu}^{g} \eta^{\mu \nu}=0$, it simply follows that the full all-loop graviton soft behavior is given by the soft theorem:

$$
\mathcal{M}_{N ; g}\left(k_{i} ; q\right)=\kappa_{D} \varepsilon_{q \mu \nu}^{S} \sum_{i=1}^{N}\left[\frac{k_{i}^{\mu} k_{i}^{\nu}}{k_{i} q}-i \frac{k_{i}^{\mu} q_{\rho}}{k_{i} q} L_{i}^{\nu \rho}-\frac{q_{\rho} q_{\sigma}}{2 k_{i} q}: L_{i}^{\mu \rho} L_{i}^{\nu \sigma}:\right] \mathcal{M}_{N}\left(k_{i}\right)+\mathcal{O}\left(q^{2}\right)
$$

where $\mathcal{M}_{N}(h)$ is the full all-loop amplitude of $N$ closed string tachyons. These amplitudes are, of course, plagued by infrared divergences, and we are here tacitly assuming that the soft limit is taken before any infrared divergent limit. This issue will be discussed in a subsequent section.

\subsection{All-loop dilaton soft theorem}

The full amplitude of one dilaton and $N$ closed tachyons is

$$
\mathcal{M}_{N ; \phi}\left(k_{i} ; q\right)=\left.\sum_{h=0}^{\infty} M_{N ; 1}^{(h)}\left(k_{i} ; q\right)\right|_{\varepsilon_{q \mu \nu}^{S}=\varepsilon_{\mu \nu}^{\phi}}
$$


where $\varepsilon_{\mu \nu}^{\phi}=\frac{1}{\sqrt{D-2}}\left(\eta_{\mu \nu}-q_{\mu} \bar{q}_{\nu}-q_{\nu} \bar{q}_{\mu}\right)$ with $\bar{q}^{2}=0$ and $q \cdot \bar{q}=1$. After contracting with this projection tensor, the $h$-loop soft behavior for the dilaton becomes:

$$
\begin{aligned}
M_{N ; \phi}^{(h)}\left(k_{i} ; q\right)= & \frac{\kappa_{D}}{\sqrt{D-2}}\left[-\sum_{i=1}^{N} \frac{m^{2}}{k_{i} q} \mathrm{e}^{q \partial_{k_{i}}}+2-\sum_{i=1}^{N} \hat{D}_{i}+h(D-2)+q_{\mu} \sum_{i=1}^{N} \hat{K}_{i}^{\mu}\right] M_{N}^{(h)} \\
& +\frac{\kappa_{D}}{2 \pi} \sqrt{D-2}\left(M_{N}^{(h)} * S_{2}^{(1)}\right)+\mathcal{O}\left(q^{2}\right)
\end{aligned}
$$

where in the case of tachyons $m^{2}=-\frac{4}{\alpha^{\prime}}$ and

$$
\hat{D}_{i}=k_{i} \cdot \frac{\partial}{\partial k_{i}}, \quad \hat{K}_{i}^{\mu}=\frac{1}{2} k_{i}^{\mu} \frac{\partial^{2}}{\partial k_{i \nu} \partial k_{i}^{\nu}}-k_{i}^{\rho} \frac{\partial^{2}}{\partial k_{i}^{\rho} \partial k_{i \mu}},
$$

which are the momentum space generators of space-time dilatations and special conformal transformations. The last term in eq. (3.16), which we have not been able to evaluate at the multiloop level, is of order $q$ and could potentially break the factorizing soft behavior of the amplitude at this order. At one-loop order, however, it turns out as explained earlier (see also appendix F) that it vanishes. Thus at least at one loop we have a soft theorem for the dilaton through subsubleading order, reading:

$$
M_{N ; \phi}^{(1)}\left(k_{i} ; q\right)=\frac{\kappa_{D}}{\sqrt{D-2}}\left[-\sum_{i=1}^{N} \frac{m^{2}}{k_{i} q} \mathrm{e}^{q \partial_{k_{i}}}+D-\sum_{i=1}^{N} \hat{D}_{i}+q_{\mu} \sum_{i=1}^{N} \hat{K}_{i}^{\mu}\right] M_{N}^{(1)}+\mathcal{O}\left(q^{2}\right) .
$$

We cannot immediately write the all-loop soft behavior in this case, because of the explicit dependence on $h$ in the dilaton soft operator. However, notice that the $h$-loop amplitude has the following scaling property

$$
M_{N}^{(h)}=\sqrt{\alpha^{\prime}}(2-D) h-2 \kappa_{D}^{2(h-1)+N} F\left(\sqrt{\alpha^{\prime}} k_{i}, R / \sqrt{\alpha^{\prime}}\right)
$$

where $F$ is a dimensionless function parametrizing the amplitude, and we recall that $R$ is denoting the compactification radii, which enter only for $h>0$. The gravitational constant is given in terms of $\alpha^{\prime}, g_{s}$, the string coupling constant, and $R$, as follows (see appendix A for a derivation):

$$
\kappa_{D}=(2 \pi)^{\frac{D-3}{2}} \sqrt{2^{-9}} g_{s}{\sqrt{\alpha^{\prime}}}^{\frac{D-2}{2}}\left(\frac{\sqrt{\alpha^{\prime}}}{R}\right)^{\frac{26-D}{2}} .
$$

From these expressions we deduce that

$$
\left[2-\sum_{i=1}^{N} k_{i} \cdot \frac{\partial}{\partial k_{i}}+(D-2) h\right] M_{N}^{(h)}=\left[\frac{D-2}{2} g_{s} \frac{\partial}{\partial g_{s}}-\sqrt{\alpha^{\prime}} \frac{\partial}{\partial \sqrt{\alpha^{\prime}}}-R \frac{\partial}{\partial R}\right] M_{N}^{(h)} .
$$

The left-hand side is nothing but the subleading $h$-loop soft dilaton operator. It is exactly reproduced by the operator on the right-hand side, which is $h$-independent. Notice also that the operator on the right-hand side leaves $\kappa_{D}$ invariant. For $h=0$, the $R$-dependence is only in $\kappa_{D}$, why in that case one can rewrite the soft operator in terms of a $D$-dimensional string coupling constant and $\alpha^{\prime}$; see eq. (A.18), which explains previous tree-level result 
where the additional $R$-operator did not appear. Slightly more explicit considerations on this soft operator are offered in appendix A, where a general 26 - D-dimensional toroidal compactification is considered.

Now we can sum all loop contributions to form the full amplitude on the right-hand side of the soft theorem, yielding through subleading order

$$
\mathcal{M}_{N ; \phi}\left(k_{i} ; q\right)=\frac{\kappa_{D}}{\sqrt{D-2}}\left[-\sum_{i=1}^{N} \frac{m^{2}}{k_{i} q} \mathrm{e}^{q \partial_{k_{i}}}+\frac{D-2}{2} g_{s} \frac{\partial}{\partial g_{s}}-\sqrt{\alpha^{\prime}} \frac{\partial}{\partial \sqrt{\alpha^{\prime}}}-R \frac{\partial}{\partial R}\right] \mathcal{M}_{N}\left(k_{i}\right)+\mathcal{O}(q)
$$

This gives the all-loop dilaton soft behavior through subleading order when scattering with $N$ closed tachyons. The same discussion about infrared divergences mentioned in the graviton case applies also here.

\section{Infrared divergences}

In the previous section we have shown that the graviton and dilaton satisfy soft factorization theorems at $h$ loops, but we have not taken into account that actually the multiloop amplitudes in the bosonic string are infrared divergent. In this section we discuss how to treat them preserving the results that we have already obtained.

In the bosonic string, where we compactify $(26-D)$ dimensions leaving $D$ non-compact dimensions, we have two kinds of infrared divergences: the first kind, appearing for any value of $D$, arises due to the fact that the bosonic string has a tachyon and a non-vanishing dilaton tadpole. As we shall see, this kind of infrared divergence does not depend on the number of external legs. When massless states are involved, another kind of infrared divergence may appear when we approach low values of non-compact dimensions, for instance when $D=4$, which from field theory are known as soft and collinear divergences. These instead depend on the number of external legs, which we shall also briefly discuss from the string theory perspective.

String amplitudes involving external tachyons, or more generally involving massive external states are also plagued by additional divergences that require mass-renormalization. As explained in ref. [72], these divergences can be regularized by not allowing the KobaNielsen variables to get too close to each other in certain configurations (see also ref. [73], ${ }^{5}$ and the recent progress in refs. [74-76]). But, since they depend only on the number of external massive legs, we do not expect that they will modify the soft operator.

Let us discuss the first kind of infrared divergences in the simplified case of the $\mathrm{N}$ tachyon amplitude at one loop, which is given by eq. (3.1b) for the case $h=1$. After explicitly deriving the $h=1$ expressions, setting $z_{i}=e^{2 \pi i \nu_{i}}$, and defining $\nu_{i j}=\nu_{i}-\nu_{j}$, the

\footnotetext{
${ }^{5}$ And references therein.
} 
one-loop $N$-tachyon amplitude reads:

$$
\begin{aligned}
T_{N}^{(1)}= & C_{1} N_{0}^{N} \int_{\mathcal{F}} d^{2} \tau \mu(\tau, \bar{\tau}) \prod_{i=1}^{N-1}\left[\int d^{2} \nu_{i}\right] \\
& \times \prod_{i<j}\left|\frac{\sin \pi \nu_{i j}}{\pi} \prod_{n=1}^{\infty} \frac{\left(1-\kappa^{n} \mathrm{e}^{2 \pi i \nu_{i j}}\right)\left(1-\kappa^{n} \mathrm{e}^{-2 \pi i \nu_{i j}}\right)}{\left(1-\kappa^{n}\right)^{2}} \mathrm{e}^{-\pi \frac{\left(\operatorname{Im} \nu_{i j}\right)^{2}}{\operatorname{Im} \tau}}\right|^{\alpha^{\prime} k_{i} k_{j}},
\end{aligned}
$$

where we have set $\eta=0, \xi=\infty, \nu_{N}=0, \kappa=\mathrm{e}^{2 \pi i \tau} . \mathcal{F}$ denotes the fundamental integration region of $\tau$, and

$$
\mu(\tau, \bar{\tau})=(2 \pi)^{2} \mathrm{e}^{4 \pi \operatorname{Im} \tau} \prod_{n=1}^{\infty}\left[\frac{1}{\left|1-\mathrm{e}^{2 \pi i \tau n}\right|^{48}}\right] \frac{(F(\tau, \bar{\tau}))^{26-D}}{(\operatorname{Im} \tau)^{D / 2}} .
$$

We now consider the region of the moduli space where all $\nu_{i}$ are very close to each other and to $\nu_{N}=0$. This can be done by introducing the variables $\eta_{i}, \varepsilon, \phi$ as follows:

$$
\mathrm{e}^{i \phi} \varepsilon \eta_{i}=\nu_{i}, i=1 \ldots N-2 ; \quad \varepsilon \mathrm{e}^{i \phi}=\nu_{N-1} ; \quad \eta_{N-1}=1 .
$$

By using these variables and keeping only the terms divergent for $\varepsilon \rightarrow 0$ we find

$$
\begin{aligned}
T_{n}^{(1)}= & C_{1} N_{0}^{N} \int_{\mathcal{F}} d^{2} \tau \mu(\tau, \bar{\tau}) \prod_{i=1}^{N-2} \int d^{2} \eta_{i} \int_{0}^{2 \pi} d \phi \int_{0}^{1} \frac{d \varepsilon}{\varepsilon^{3-\frac{\alpha^{\prime}}{2} p^{2}}} \prod_{i<j}\left|\eta_{i j}\right|^{\alpha^{\prime} k_{i} k_{j}} \\
& \times\left[1-\alpha^{\prime} \sum_{i<j} k_{i} k_{j} \frac{\pi \varepsilon^{2}}{\operatorname{Im} \tau}\left(\sin \phi \operatorname{Re}\left(\eta_{i j}\right)+\cos \phi \operatorname{Im}\left(\eta_{i j}\right)\right)^{2}+\mathcal{O}\left(\varepsilon^{4}\right)\right],
\end{aligned}
$$

where by $\phi$-integration a second $\varepsilon^{-1}$-term was removed, and where the divergent terms for $\varepsilon \sim 0$ were regularized by the substitution $-3 \rightarrow-3+\frac{\alpha^{\prime}}{2} p^{2}$. The upper limit in the integral was set to $\varepsilon=1$, since we are interested in the behavior near $\varepsilon \sim 0$. The integrals over $\phi$ and $\varepsilon$ can be performed and one gets:

$$
\begin{aligned}
T_{N}^{(1)}= & C_{1} N_{0}^{N} \int_{\mathcal{F}} d^{2} \tau \mu(\tau, \bar{\tau}) \prod_{i=1}^{N-2} \int d^{2} \eta_{i} \prod_{i<j}\left|\eta_{i j}\right|^{\alpha^{\prime} k_{i} k_{j}} \\
& \times\left[-\frac{2 \pi}{2-\frac{\alpha^{\prime}}{2} p^{2}}+\frac{2 \pi}{p^{2}} \frac{\pi}{\operatorname{Im} \tau} \sum_{i<j} k_{i} k_{j} \eta_{i} \bar{\eta}_{j}\right]+\cdots,
\end{aligned}
$$

where the dots denote terms that are regular for $p^{2} \rightarrow 0$. The first term in the last line corresponds to the regularized tachyon contribution, while the second term, corresponding to the dilaton contribution, is divergent when $p^{2} \rightarrow 0$. The second term in the square bracket has been obtained by using the identity:

$$
\sum_{i<j} k_{i} k_{j}\left|\eta_{i}-\eta_{j}\right|^{2}=\frac{1}{2} \sum_{i, j=1}^{N} k_{i} k_{j}\left|\eta_{i}-\eta_{j}\right|^{2}=-\sum_{i, j=1}^{N} k_{i} k_{j} \eta_{i} \bar{\eta}_{j}
$$

which follows from momentum conservation for $p \sim 0$. 
Finally, we recognise that the coefficients of the two poles are the tree-level amplitude with $(N+1)$ tachyons and that with $N$ tachyons and one dilaton that we rewrite here:

$$
\begin{aligned}
T_{(N+1) \operatorname{tach}} & =C_{0} N_{0}^{N+1} \prod_{i=1}^{N-2} \int d^{2} \eta_{i} \prod_{i<j=1}^{N}\left|\eta_{i}-\eta_{j}\right|^{\alpha^{\prime} k_{i} k_{j}} \\
T_{N \text { tach }+1 \text { dil }} & =C_{0} N_{0}^{N+1} \frac{\alpha^{\prime}}{2} \prod_{i=1}^{N-2} \int d^{2} \eta_{i} \prod_{i<j=1}^{N}\left|\eta_{i}-\eta_{j}\right|^{\alpha^{\prime} k_{i} k_{j}} \sum_{i, j=1}^{N} k_{i}^{\mu} k_{j}^{\nu} \eta_{i} \bar{\eta}_{j} .
\end{aligned}
$$

Using them and the fact that $p \sim 0$ we can rewrite eq. (4.6) as follows [69, 70]:

$$
T_{N}^{(1)}=\frac{C_{1}}{C_{0} N_{0}} \int_{\mathcal{F}} d^{2} \tau \mu(\tau, \bar{\tau})\left[-\frac{2 \pi T_{(N+1) \operatorname{tach}}}{2-\frac{\alpha^{\prime}}{2} p^{2}}+\frac{(2 \pi)^{2}}{\alpha^{\prime} p^{2} \operatorname{Im} \tau} T_{N \text { tach }+1 \mathrm{dil}}\right]+\cdots
$$

In conclusion, we get the first term in the square bracket in eq. (4.8) that has the propagator of the tachyon times the amplitude with $(N+1)$ tachyons with one of them connected to the closed string tadpole that has to be regularized for large values of $\operatorname{Im} \tau$. The introduction of the finite momentum $p$ has regularized the contribution of the tachyon propagator. The second term in the square bracket has the pole of the massless dilaton attached to the closed string tadpole, times the amplitude with $N$ tachyons and one dilaton. This term is divergent when $p \rightarrow 0$, but can be regularized keeping $p \neq 0$.

In conclusion, we have shown that, in a certain region of the moduli space the bosonic string exhibits the divergence due to the dilaton tadpole. We have regularized it by introducing an infrared cutoff $p$. As already mentioned, another cutoff for $\operatorname{Im} \tau \rightarrow \infty$ should also be introduced to regularize the closed string tadpole. These divergences are, however, present in both the amplitude with $(N+1)$ particles and in that with $N$ particles that appear in the soft theorem. Therefore, by regularizing both of them in the same way with an infrared cutoff, the soft theorems found in the previous section are still satisfied.

If we take the number of non-compact directions down to $D=4$, then one gets additional infrared divergences. They are obtained in the $\operatorname{limit} \operatorname{Im} \tau \rightarrow \infty$ and are discussed in detail in ref. [71] for the closed superstring for amplitudes with massless states. They correspond to the soft infrared divergences that one also finds in field theory that, depending on the number of external legs, prevents the soft theorem, found at the tree level, to be also valid at loop level [52]. In field theory, one also encounter collinear divergences in certain Feynman loop diagrams of massless amplitudes. However, it is a time-honored result due to Weinberg [77], which has since been proven to all orders in perturbation theory [78], that such divergence do not appear in the full amplitude involving gravitons and other massless states. We will not consider these divergences in more detail in this paper and refer to ref. [71] for the interested reader (see also ref. [79] for a discussion on these divergences in the classical limit).

In conclusion, the multiloop soft theorem found in this paper is valid when the number of non-compact dimensions is greater than four. 


\section{Conclusions}

In this paper we have extended to multiloops in the bosonic string the soft theorems for the graviton and the dilaton. This has been done by computing the amplitude with one graviton/dilaton and $N$ tachyons and by explicitly studying its behaviour in the limit where the massless states carry low momentum. The main ingredient used to compute this amplitude to an arbitrary order of the perturbative expansion has been the $h$-loop $N$-Reggeon vertex. This is an operator, constructed in the $80 \mathrm{~s}$, that when applied on an arbitrary number of external states, not necessarily on-shell, provides the scattering amplitudes corresponding to the external states taken in consideration.

The amplitude that we have computed, as any loop amplitude in the bosonic string, suffers from infrared (IR) divergences. They appear in different corners of the moduli space and in principle may modify the tree-level soft operators. One corner of moduli space that leads to these IR divergences corresponds to a world-sheet topology where one or more loops, tadpoles, are connected by long tubes to a sphere where all the external states are inserted. The integration over the modulus parametrizing the length of this tube gives rise to divergences which are due to the exchange between the loops and the sphere of tachyons and on-shell dilatons. We have regularized these divergences by introducing a finite momentum for the intermediate states. Furthermore we have regularized the closed string tadpole by introducing a cut-off for large values of the string moduli. These regularizations, being independent on the number of external states, do not affect soft theorems.

Another class of IR divergences appears when string theories are compactified down to four dimensions. They have been studied in literature [71] and the main feature is that they depend on the number of the external states and therefore affect the soft theorems. They can be avoided by compactifying string amplitudes down to a space-time dimension $D>4$ [79]. Under such a limitation and for amplitudes with one soft graviton/dilaton and $N$ hard tachyons the main result of our analysis is that the soft graviton behaviour at loop level coincides with the one at the tree level. This happens because the loop amplitudes have the same form, in terms of the Green function, as the tree-level ones, except for an extra contribution in the case of the dilaton which is importantly there to ensure the right scaling relation at every loop order.

It is by now well established that tree-level soft theorems of gauge and gravitational fields are a consequence of the gauge symmetries underlying the theory. The IR behaviour of an amplitude, $M_{N+1}=\varepsilon_{\mu \nu} M_{N+1}^{\mu \nu}$, with a graviton carrying low momentum $q$ and $N$ hard particles is fully determined, up to the order $q$, by the gauge invariance conditions:

$$
q_{\mu}\left(M_{N+1}^{\mu \nu}\left(q,\left\{k_{i}\right\}\right)-f\left(q,\left\{k_{i}\right\}\right) \eta^{\mu \nu}\right)=q_{\nu}\left(M_{N+1}^{\mu \nu}\left(q,\left\{k_{i}\right\}\right)-f\left(q,\left\{k_{i}\right\}\right) \eta^{\mu \nu}\right)=0 .
$$

Here $f\left(q,\left\{k_{i}\right\}\right)$ is an arbitrary function of the momenta that contributes only in the case of a soft dilaton. Its arbitrariness prevent us to get the full soft behaviour of the dilaton from gauge invariance. In string theory this quantity can be explicitly computed and from ref. [70] it is well known that it differs from zero in the presence of massless open string states. In this paper we have seen that it also differs from zero at the loop level of only closed strings but that the soft behaviour of multiloop amplitudes with only closed string 
states can be obtained from gauge invariance by taking this function equal to the number of handles, $h$, of the Riemann surface, at least through subleading order. This choice does not affect the tree-level gauge conditions but the loop ones are modified in such a way to reproduce exactly the extra contribution given in eq. (3.6). At the level of the full amplitude, the extra contribution at loop-level singles out the scaling operator

$$
\frac{D-2}{2} g_{s} \frac{\partial}{\partial g_{s}}-\sqrt{\alpha^{\prime}} \frac{\partial}{\partial \sqrt{\alpha^{\prime}}}-R \frac{\partial}{\partial R},
$$

as the proper multiloop dilatation operator determining the order $q^{0}$ soft behavior of the dilaton. Here $R$ denotes collectively the compactification radii.

It is not clear yet if the tree-level universality of the soft dilaton theorem, i.e. its independence of the string theory considered, extends to loops. Indeed this paper, in a special case, shows that the multiloop soft dilaton operator, when written in terms of the string slope, string coupling constant, and compactification parameters, is the same as at tree-level at least through subleading order, and at one loop we could show that they are the same even through subsubleading order. It remains an open problem to understand whether the subsubleading one loop behavior extends to all loops, and it would be very interesting to explore its universality at loop level in other string theories and for arbitrary hard states.

\section{Acknowledgments}

We thank Igor Pesando and Rodolfo Russo for many useful discussions. We additionally thank Rodolfo Russo, and Stefano Sciuto for a critical reading of the manuscript. We also would like to thank the referee of this paper and Massimo Bianchi for, respectively in the referee report and in a seminar, pointing out to us that the dependence on the compactification radii was not fully taken into account in the formulation of the dilaton soft theorem that appeared in the first version of the paper.

\section{A On the gravitational coupling constant in string theory}

For both the bosonic string, in the critical $d=26$ dimensions, and the superstring, in $d=10$, one can derive the following formula for the $D p$-brane tension $T_{p}[80,81]$ :

$$
T_{p}=\frac{\sqrt{\pi}}{2^{\frac{d-10}{4}}}\left(2 \pi \sqrt{\alpha^{\prime}}\right)^{\frac{d}{2}-p-2} ; \quad \tau_{p} \equiv \frac{T_{p}}{\kappa_{d}}
$$

where $\tau_{p}$ is the physical tension of a Dp-brane. In eq. (13.3.20) of ref. [81] it is proposed that the string coupling constant $g_{s}$ is given by the ratio of the fundamental string tension $\tau_{F 1}=\frac{1}{2 \pi \alpha^{\prime}}$ and the tension $\tau_{D 1}$ of the D1-brane:

$$
\frac{\tau_{F 1}}{\tau_{D 1}}=g_{s}=\frac{\kappa_{d}}{2 \pi \alpha^{\prime}} \frac{2^{\frac{d-10}{4}}}{\sqrt{\pi}\left(2 \pi \sqrt{\alpha^{\prime}}\right)^{\frac{d}{2}-3}}
$$

It implies

$$
2 \kappa_{d}^{2}=\frac{g_{s}^{2}(2 \pi)^{d-3}\left(\sqrt{\alpha^{\prime}}\right)^{d-2}}{2^{\frac{d-10}{2}}}
$$


that for the bosonic and superstring respectively gives:

$$
2 \kappa_{26}^{2}=2^{-8} g_{s}^{2}(2 \pi)^{23}\left(\alpha^{\prime}\right)^{12} ; \quad 2 \kappa_{10}^{2}=g_{s}^{2}(2 \pi)^{7}\left(\alpha^{\prime}\right)^{4}
$$

We consider in the following the compactification of the effective action of the bosonic string theory, where $d=26$. The analysis, however, can be equally performed in the $d=10$ superstring theory case. The terms of the effective action of the bosonic string theory, in the string frame, relevant for the forthcoming discussion are:

$$
S=\frac{\mathrm{e}^{-2 \hat{\phi}_{0}}}{2 \hat{\kappa}^{2}} \int d^{26} \hat{x} \sqrt{|\hat{G}|} \mathrm{e}^{-2 \hat{\phi}}(\hat{R}(\hat{x})+\ldots) ; \quad \hat{\phi}=\phi_{26}-\hat{\phi}_{0}
$$

where

$$
2 \hat{\kappa}^{2}=2^{-8}(2 \pi)^{23}\left(\alpha^{\prime}\right)^{12}=2\left(\kappa_{26} / g_{s}\right)^{2}
$$

Compactifying $26-D$-dimensions, the 26 -dimensional Lorentz group $\mathrm{SO}(1,25)$ is broken to $\mathrm{SO}(1, D-1) \times \mathrm{SO}(26-D)$. We hence decompose the space-time coordinates into $\hat{x}^{M}=\left(x^{\mu}, y^{m}\right)$ with $\mu=0 \ldots D-1$ and $m=D \ldots 25$. Under the assumption that the 26-dimensional metric, $\hat{G}_{M N}$, admits the following block-diagonal form

$$
\hat{G}_{M N}=\left(\begin{array}{cc}
g_{\mu \nu}\left(x^{\mu}\right) & 0 \\
0 & G_{m n}\left(x^{\mu}\right)
\end{array}\right) ; \quad M, N=0 \ldots 25
$$

the low-energy string action can be reduced to an effective $D$-dimensional action, $S_{D}$, as follows: first, under eq. (A.7), the action reduces to

$$
S_{D}=\frac{\mathrm{e}^{-2 \hat{\phi}_{0}}}{2 \hat{\kappa}^{2}} \int d^{D} x \sqrt{|g|} \int_{0}^{2 \pi \sqrt{\alpha^{\prime}}} d^{26-D} y \sqrt{G} e^{-2 \hat{\phi}}(\hat{R}(x)+\ldots)
$$

Next, introduce the $D$-dimensional dilaton, $\phi(x)$, through:

$$
\mathrm{e}^{-2 \hat{\phi}_{0}} \sqrt{G} e^{-2 \hat{\phi}} \equiv \mathrm{e}^{-2 \phi} \mathrm{e}^{-2 \phi_{0}}
$$

where

$$
\mathrm{e}^{-2 \phi}=\mathrm{e}^{-2 \hat{\phi}} \frac{\sqrt{G}}{\sqrt{G_{0}}} ; \quad \mathrm{e}^{-2 \phi_{0}}=\mathrm{e}^{-2 \hat{\phi}_{0}} \sqrt{G_{0}},
$$

and $G_{0}$ denotes the vacuum expectation value of the compact metric. Taking the compact manifold to be the product of $26-D$ circles with radii $R_{m}$, we get

$$
\sqrt{G_{0}}=\frac{\prod_{m=1}^{26-D} R_{m}}{\left(\sqrt{\alpha^{\prime}}\right)^{26-D}}
$$

With these ingredients, eq. (A.8) becomes:

$$
\begin{aligned}
S_{D} & =\frac{\left(2 \pi \sqrt{\alpha^{\prime}}\right)^{26-D} \mathrm{e}^{-2 \phi_{0}}}{2 \hat{\kappa}^{2}} \int d^{D} x \sqrt{|g|} \mathrm{e}^{-2 \phi}(\hat{R}(x)+\ldots) \\
& =\frac{1}{2 \kappa_{D}^{2}} \int d^{D} x \sqrt{|g|} \mathrm{e}^{-2 \phi}(\hat{R}(x)+\ldots)
\end{aligned}
$$


where we note that $\hat{R}(x)$ is the Ricci scalar computed from the 26-dimensional metric, eq. (A.7), which, however, only depends on the $D$-dimensional noncompact coordinates $x^{\mu}$. It follows that

$$
\frac{1}{2 \kappa_{D}^{2}}=\frac{\left(2 \pi \sqrt{\alpha^{\prime}}\right)^{26-D} \mathrm{e}^{-2 \hat{\phi}_{0}} \sqrt{G_{0}}}{2 \hat{\kappa}^{2}}=\left(\frac{g_{s}^{2}}{\mathrm{e}^{2 \hat{\phi}_{0}}}\right) \frac{\prod_{m=1}^{26-D}\left(2 \pi R_{m}\right)}{2 \kappa_{26}^{2}} .
$$

Following conventions, we set $g_{s}=\mathrm{e}^{\hat{\phi}_{0}}$, whereby the prefactor above is simply one. Inserting the explicit expression from eq. (A.4) for $\kappa_{26}$ we get

$$
\kappa_{D}=\frac{g_{s}(2 \pi)^{23 / 2}\left(\alpha^{\prime}\right)^{6}}{\sqrt{2}^{9} \prod_{m=1}^{26-D} \sqrt{2 \pi R_{m}}}=(2 \pi)^{\frac{D-3}{2}} \sqrt{2^{-9}} g_{s} \sqrt{\alpha^{\prime}} \frac{D-2}{2} \prod_{m=1}^{26-D}\left(\frac{\sqrt{\alpha^{\prime}}}{R_{m}}\right)^{\frac{1}{2}}
$$

It is easy to check that $\kappa_{D}$ satisfies the following identity:

$$
\left(\frac{D-2}{2} g_{s} \frac{\partial}{\partial g_{s}}-\sqrt{\alpha^{\prime}} \frac{\partial}{\partial \sqrt{\alpha^{\prime}}}-\sum_{m=1}^{26-D} R_{m} \frac{\partial}{\partial R_{m}}\right) \kappa_{D}=0 .
$$

The $R_{m}$ dependence can also be absorbed by introducing the $D$-dimensional string tension $g_{D}[82]$ :

$$
g_{D} \equiv \mathrm{e}^{\phi_{0}}=g_{s} G_{0}^{-\frac{1}{4}}
$$

Then eq. (A.13) becomes:

$$
\frac{1}{2 \kappa_{D}^{2}}=\frac{\left(2 \pi \sqrt{\alpha^{\prime}}\right)^{26-D}}{2 g_{D}^{2} \hat{\kappa}^{2}} ; \quad \kappa_{D}=(2 \pi)^{\frac{D-3}{2}} \sqrt{2^{-9}} g_{D}\left(\sqrt{\alpha^{\prime}}\right)^{\frac{D-2}{2}} .
$$

and thus $\kappa_{D}$ also satisfies

$$
\left(\frac{D-2}{2} g_{D} \frac{\partial}{\partial g_{D}}-\sqrt{\alpha^{\prime}} \frac{\partial}{\partial \sqrt{\alpha^{\prime}}}\right) \kappa_{D}=0
$$

\section{B Schottky parametrization of Riemann surfaces}

In this appendix we summarize the main aspects of the Schottky description of Riemann surfaces of arbitrary genus. String amplitudes at $h$-order in the perturbative expansion are represented by integrals over Riemann surfaces, $\Sigma_{h}$, of genus $h$. The world sheet of closed string tree diagrams, for example, is a sphere which is mapped by a stereographic projection onto $\mathbb{C} P^{1}$, the complex plane with the point at infinity. This complex plane is the integration region of tree-level closed string amplitudes. Riemann surfaces of higher order $\Sigma_{h}$, in the Schottky representation, are essentially identified with the complex plane where $2 h$ cycles have been cut-off and pairwise identified.

\section{B.1 The Schottky group}

The basic element of the Schottky group is the projective transformation which maps $\mathbb{C} P^{1}$ to itself. It can be expressed by a matrix multiplication [83]:

$$
S=\left(\begin{array}{ll}
a & b \\
c & d
\end{array}\right) ; \quad S:\left(\begin{array}{l}
z_{1} \\
z_{2}
\end{array}\right) \rightarrow\left(\begin{array}{l}
z_{1}^{\prime} \\
z_{2}^{\prime}
\end{array}\right)=\left(\begin{array}{ll}
a & b \\
c & d
\end{array}\right)\left(\begin{array}{l}
z_{1} \\
z_{2}
\end{array}\right)
$$


The entries of the matrix are complex numbers with $a d-b c=1$. The vectors ${ }^{6}\left(z_{1}, z_{2}\right)^{t} \in$ $\mathbb{C}^{2}$ are the homogeneous coordinates of points of $\mathbb{C} P^{1}$. For $z_{2} \neq 0$ and $z_{2}^{\prime} \neq 0$ we can define $z=z_{1} / z_{2}$ and $z^{\prime}=z_{1}^{\prime} / z_{2}^{\prime}$ and rewrite the projective transformation in the form:

$$
z^{\prime}=\frac{a z+b}{c z+d} .
$$

Such a rational mapping is called a Möbius transformation. A property of the projective transformation is to leave invariant the cross ratio of four points:

$$
\frac{\left(z_{1}^{\prime}-z_{2}^{\prime}\right)\left(z_{3}^{\prime}-z_{4}^{\prime}\right)}{\left(z_{1}^{\prime}-z_{3}^{\prime}\right)\left(z_{2}^{\prime}-z_{4}^{\prime}\right)}=\frac{\left(z_{1}-z_{2}\right)\left(z_{3}-z_{4}\right)}{\left(z_{1}-z_{3}\right)\left(z_{2}-z_{4}\right)}
$$

The fixed points $\xi$ and $\eta$ are by definition the points left invariant by the transformation (B.2). These two points together with the point $\infty$ are mapped into $\xi, \eta$ and $a / c$ respectively. Hence eq. (B.3) for $\left(z_{2}=\eta, z_{3}=\xi, z_{4}=\infty\right)$ may be equivalently written as:

$$
\frac{S(z)-\eta}{S(z)-\xi}=\kappa \frac{z-\eta}{z-\xi} ; \quad \kappa=\frac{a-c \eta}{a-c \xi} ; \quad|\kappa| \leq 1
$$

where $\kappa$ is the multiplier of the projective transformation $S(z)$ and it is easily seen that $\kappa^{n}$, with $n$ a positive integer, is the multiplier of the $S^{n}(z)$ transformation. It follows that:

$$
\lim _{n \rightarrow \infty} S^{n}(z)=\eta, \quad \lim _{n \rightarrow \infty} S^{-n}(z)=\xi
$$

$\eta$ and $\xi$ are named attractive and repulsive fixed points, respectively. The infinitesimal line element $d z$ is transformed by $S(z)$ in:

$$
\frac{d z^{\prime}}{d z}=S^{\prime}(z)=\frac{1}{(c z+d)^{2}}
$$

Lengths and areas are unaltered in magnitude if $|c z+d|=1$ For $c \neq 0$ the locus is a circle of radius $1 /|c|$ and center $-d / c$. This circle is called the isometric circle $\mathcal{C}$. Similar considerations can be done for the inverse transformation $S^{-1}(z)$. In this case the isometric circle, $\mathcal{C}^{\prime}$, is $|c z-a|=1$ and it has radius $1 /|c|$ and center $a / c$. It is easily seen that

$$
|c S(z)-a|=|c z+d|^{-1}
$$

therefore $S(z)$ maps $\mathcal{C} \rightarrow \mathcal{C}^{\prime}$ while the inverse of the transformation, $S^{-1}(z)$, transforms $\mathcal{C}^{\prime}$ into $\mathcal{C}$. Moreover any point inside $\mathcal{C}$ is transformed by the projective transformation in a point outside the circle $\mathcal{C}^{\prime}$ and, vice versa, the region exterior to $\mathcal{C}^{\prime}$ is mapped by $S^{-1}$ in the interior of $\mathcal{C}$. In particular $\eta$ will be in the circle $\mathcal{C}^{\prime}$ and $\xi$ in the other isometric circle $[59,84]$.

We can now introduce the definition of Schottky group. Given a set of $h$ projective transformations $S_{I}$, where $I=(1 \ldots h)$, with the restriction that all isometric circles are external to each other and therefore have zero intersection, the Schottky group, $\mathscr{C}_{h}$, is the group freely generated by the $S_{I}$ 's $[59,84]$. A generic element of the group $T_{\alpha} \in \mathscr{C}_{h}$, except for the identity, can be written in the form:

$$
T_{\alpha}=S_{I_{1}}^{n_{1}} S_{I_{2}}^{n_{2}} \ldots S_{I_{r}}^{n_{r}}, \quad r=1,2 \ldots ; \quad n_{i} \in \mathbb{Z} /\{0\}, \quad I_{i} \neq I_{i+1}
$$

\footnotetext{
${ }^{6}$ Here $t$ denotes the transpose of the vector.
} 
The number of the generators or their inverse gives the order of the group:

$$
n_{\alpha}=\sum_{i=1}^{r}\left|n_{r}\right|
$$

$T_{\alpha}$ is a primitive element of the Schottky group if it cannot be written as an integer power of other elements, $T_{\alpha} \neq\left(T_{\alpha^{\prime}}\right)^{n}$ with $n \in \mathbb{N}_{+}$. The fundamental region of the group is the complex plane outside all the isometric $\operatorname{circles} \mathcal{C}_{I}$ and $\mathcal{C}_{I}^{\prime}$ associated to each projective transformation defining the group. By identifying $\mathcal{C}_{I}$ with $\mathcal{C}_{I}^{\prime}, \forall I=(1 \ldots h), h$ handles are formed and a Riemann surface of genus $h$ is created. More precisely:

$$
\Sigma_{h}=\frac{\mathbb{C} \cup\{\infty\}-\Lambda\left(\mathscr{C}_{h}\right)}{\mathscr{C}_{h}}
$$

where $\Lambda\left(\mathscr{C}_{h}\right)$ is the limit set of the Schottky group [59, 85]. Equivalent representations of the Schottky group are obtained by transforming each element by a fixed projective transformation $A$, i.e. $T_{\alpha}^{\prime}=A T_{\alpha} A^{-1}$. Each generator of the group depends on three complex parameters, the fixed points and the multiplier, but three of them can be fixed by this overall projective transformation, therefore inequivalent Schottky groups are parametrized by $3 h-3$ complex numbers. This is exactly the number of complex moduli of a Riemann surface with $h$ handles.

\section{B.2 The Abelian differentials and the period matrix}

The canonical homology cycles $\left(a_{I}, b_{I}\right)$ of a Riemann surface are identified respectively with $\mathcal{C}_{I}$ or $\mathcal{C}_{I}^{\prime}$ and with the path connecting a point $z_{0} \in \mathcal{C}_{I}$ with $S_{I}\left(z_{0}\right) \in \mathcal{C}^{\prime}$.

The abelian differentials $\omega_{I}, I=(1 \ldots h)$, associated to the homology cycles are given by:

$$
\omega_{I}=\sum_{T_{\alpha}}^{(I)}\left(\frac{1}{z-T_{\alpha}\left(\eta_{I}\right)}-\frac{1}{z-T_{\alpha}\left(\xi_{I}\right)}\right) d z \equiv \omega_{I}(z) d z
$$

where $\sum_{T_{\alpha}}^{(I)}$ means that the sum is over all the elements of the Schottky group that do not have $S_{I}^{n}, n \in \mathbb{Z} /\{0\}$ at their right-hand end. The abelian differentials are normalized in the standard way:

$$
\oint_{a_{J}} \omega_{I}=\oint_{\mathcal{C}_{J}^{\prime}} \omega_{I}=2 \pi i \delta_{I J}
$$

The normalization follows from the observation that the abelian differentials have simple poles in $T_{\alpha}\left(\eta_{I}\right)$ and $T_{\alpha}\left(\xi_{I}\right)$ and these two points are always outside the circle $a_{J}$ except when $S_{J}$ appears as leftmost factor in $T_{\alpha}$. In this case they are both inside the same circle $\mathcal{C}_{J}^{\prime}$ and the integral along this circle is vanishing from the residue theorem. When $T_{\alpha}$ is the identity, instead, the first term of eq. (B.11) gives the normalization of the integral while the second term is vanishing after integration. The integral along the circle $b_{J}$ is by definition:

$$
\oint_{b_{J}} \omega_{I}=\int_{z_{0}}^{S_{J}\left(z_{0}\right)} \omega_{I}=\sum_{T_{\alpha}}^{(I)} \log \frac{S_{J}\left(z_{0}\right)-T_{\alpha}\left(\eta_{I}\right)}{z_{0}-T_{\alpha}\left(\eta_{I}\right)} \frac{z_{0}-T_{\alpha}\left(\xi_{I}\right)}{S_{J}\left(z_{0}\right)-T_{\alpha}\left(\xi_{I}\right)}
$$


With the help of the identity

$$
\frac{\left(S_{J}\left(z_{0}\right)-T_{\alpha}\left(\eta_{I}\right)\right)\left(z_{0}-T_{\alpha}\left(\xi_{I}\right)\right)}{\left(S_{J}\left(z_{0}\right)-T_{\alpha}\left(\xi_{I}\right)\right)\left(z_{0}-T_{\alpha}\left(\eta_{I}\right)\right)}=\frac{\left(T_{\alpha}^{-1} S_{J}\left(z_{0}\right)-\eta_{I}\right)\left(T_{\alpha}^{-1}\left(z_{0}\right)-\xi_{I}\right)}{\left(T_{\alpha}^{-1} S_{J}\left(z_{0}\right)-\xi_{I}\right)\left(T_{\alpha}^{-1}\left(z_{0}\right)-\eta_{I}\right)}
$$

the right side of the eq. (B.13) for $I \neq J$ becomes:

$$
\sum_{T_{\alpha}}^{(I)} \log \frac{\left(T_{\alpha}^{-1} S_{J}\left(z_{0}\right)-\eta_{I}\right)\left(T_{\alpha}^{-1}\left(z_{0}\right)-\xi_{I}\right)}{\left(T_{\alpha}^{-1} S_{J}\left(z_{0}\right)-\xi_{I}\right)\left(T_{\alpha}\left(z_{0}\right)-\eta_{I}\right)}=\sum_{T_{\alpha}}^{(J, I)} \sum_{n \in \mathbb{Z}} \log \frac{\left(T_{\alpha}^{-1} S_{J}^{n+1}\left(z_{0}\right)-\eta_{I}\right)\left(T_{\alpha}^{-1} S_{J}^{n}\left(z_{0}\right)-\xi_{I}\right)}{\left(T_{\alpha}^{-1} S_{J}^{n+1}\left(z_{0}\right)-\xi_{I}\right)\left(T_{\alpha} S_{J}^{n}\left(z_{0}\right)-\eta_{I}\right)}
$$

where $(J, I)$ means a sum over the elements $T_{\alpha}$ without $I$ and $J$ as rightmost and leftmost factor, respectively. It is easy to see that the sum over $n$ vanishes due to the properties of the logarithm, except at the points $n= \pm \infty$, thus getting for $I \neq J[64]$ :

$$
\begin{aligned}
\oint_{b_{J}} \omega_{I} & =\sum_{T_{\alpha}}^{(J, I)} \log \frac{\left(T_{\alpha}^{-1} S_{J}^{\infty}\left(z_{0}\right)-\eta_{I}\right)}{\left(T_{\alpha}^{-1} S_{J}^{\infty}\left(z_{0}\right)-\xi_{I}\right)} \frac{\left(T_{\alpha}^{-1} S_{J}^{-\infty}\left(z_{0}\right)-\xi_{I}\right)}{\left(T_{\alpha}^{-1} S_{J}^{-\infty}\left(z_{0}\right)-\eta_{I}\right)} \\
& =\sum_{T_{\alpha}}^{(J, I)} \log \frac{\left(\eta_{J}-T_{\alpha}\left(\eta_{I}\right)\right)}{\left(\eta_{J}-T_{\alpha}\left(\xi_{I}\right)\right)} \frac{\left(\xi_{J}-T_{\alpha}\left(\xi_{I}\right)\right)}{\left(\xi_{J}-T_{\alpha}\left(\eta_{I}\right)\right)},
\end{aligned}
$$

where $S_{J}^{\infty}\left(z_{0}\right)=\eta_{J}$ and $S^{-\infty}\left(z_{0}\right)_{J}=\xi_{J}$. The same analysis can be repeated in the case $I=J$, the only difference is that in eq. (B.15) for $n \neq 0$ the case $T_{\alpha}=\mathbb{I}$ has to be excluded because it violates the restriction that $T_{\alpha}$ doesn't have $S_{I}$ as rightmost factor. This means that, in using eq. (B.14), the identity has to be excluded, getting:

$$
\sum_{T_{\alpha} \neq \mathbb{I}}^{(I)} \log \frac{S_{I}\left(z_{0}\right)-T_{\alpha}\left(\eta_{I}\right)}{z_{0}-T_{\alpha}\left(\eta_{I}\right)} \frac{z_{0}-T_{\alpha}\left(\xi_{I}\right)}{S_{I}\left(z_{0}\right)-T_{\alpha}\left(\xi_{I}\right)}=\sum_{T_{\alpha} \neq \mathbb{I}}^{(I, I)} \log \frac{\left(\eta_{I}-T_{\alpha}\left(\eta_{I}\right)\right)}{\left(\eta_{I}-T_{\alpha}\left(\xi_{I}\right)\right)} \frac{\left(\xi_{I}-T_{\alpha}\left(\xi_{I}\right)\right)}{\left(\xi_{I}-T_{\alpha}\left(\eta_{I}\right)\right)}
$$

The contribution from the identity is instead given by:

$$
\log \frac{S_{I}\left(z_{0}\right)-\eta_{I}}{S_{I}\left(z_{0}\right)-\xi_{I}} \frac{z_{0}-\xi_{I}}{z_{0}-\eta_{I}}=\log \kappa_{I}
$$

In conclusion we have:

$$
\oint_{b_{J}} \omega_{I}=2 \pi i \tau_{I J}
$$

where

$$
2 \pi i \tau_{I J}=\delta_{I J} \log \kappa_{I}-\sum_{T_{\alpha}}^{\prime(J, I)} \log \frac{\left(\eta_{J}-T_{\alpha}\left(\xi_{I}\right)\right)}{\left(\eta_{J}-T_{\alpha}\left(\eta_{I}\right)\right)} \frac{\left(\xi_{J}-T_{\alpha}\left(\eta_{I}\right)\right)}{\left(\xi_{J}-T_{\alpha}\left(\xi_{I}\right)\right)}
$$

and the prime on the sum means that the identity is not present for $I=J$. The quantity $\tau_{I J}$ is the period matrix in the Schottky representation of the Riemann surface.

\section{B.3 The prime form}

The prime form $\mathbb{E}\left(z_{1}, z_{2}\right)$ is a differential form with conformal weight $(-1 / 2,-1 / 2)$. In the Schottky parametrization it is given by:

$$
\mathbb{E}\left(z_{1}, z_{2}\right)=\frac{\left(z_{1}-z_{2}\right)}{\sqrt{d z_{1} d z_{2}}} \prod_{\alpha}^{\prime} \frac{\left(z_{1}-T_{\alpha}\left(z_{2}\right)\right)\left(z_{2}-T_{\alpha}\left(z_{1}\right)\right)}{\left(z_{1}-T_{\alpha}\left(z_{1}\right)\right)\left(z_{2}-T_{\alpha}\left(z_{2}\right)\right)} \equiv \frac{E\left(z_{1}, z_{2}\right)}{\sqrt{d z_{1} d z_{2}}}
$$


where the prime means that the identity is excluded and $T_{\alpha}$ and $T_{\alpha}^{-1}$ are counted only once. The prime form doesn't change if we move the argument around the $a_{I}$ cycles. It changes around the $b_{I}$ cycles according to the rule [59]:

$$
\mathbb{E}\left(S_{I}\left(z_{1}\right), z_{2}\right)=-e^{-i \pi \tau_{I I}} e^{-\int_{z_{2}}^{z_{1}} \omega_{I}} \mathbb{E}\left(z_{1}, z_{2}\right)
$$

In what follows we also give the periodicity properties of the function $E\left(z_{1}, z_{2}\right)$ around the homology cycles of the Riemann surface. This function is, by its definition, invariant around the cycle $a_{I}$, while from the identity:

$$
d S_{I}(z)=\frac{d z}{\left(c_{I} z+d_{I}\right)^{2}}
$$

we have

$$
E\left(S_{I}\left(z_{1}\right), z_{2}\right)=\mathbb{E}\left(S_{I}\left(z_{1}\right), z_{2}\right) \sqrt{d S_{I}\left(z_{1}\right) d z_{2}}=-\frac{1}{\left(c_{I} z_{1}+d_{I}\right)} e^{-i \pi \tau_{I I}} e^{-\int_{z_{2}}^{z_{1}} \omega_{I}} E\left(z_{1}, z_{2}\right)
$$

In this paper, with an abuse of notation, we call both $\mathbb{E}\left(z_{1}, z_{2}\right)$ and $E\left(z_{1}, z_{2}\right)$ the prime form, although the latter is not a form.

An alternative expression of the prime form given in terms of the Riemann Theta function is [86]: ${ }^{7}$

$$
\mathbb{E}=\frac{\Theta\left[\begin{array}{l}
\vec{\delta}_{1} \\
\vec{\delta}_{2}
\end{array}\right]\left(\vec{\nu}_{w}-\vec{\nu}_{z} \mid \tau\right)}{\sqrt{\zeta(w) d z} \sqrt{\zeta(w) d w}} ; \quad \quad \vec{\nu}_{w}-\vec{\nu}_{z}=\frac{1}{2 \pi i} \int_{z}^{w} \vec{\omega}
$$

where $\vec{\delta}_{1,2}$ are fixed odd theta characteristics, $\zeta(z)=\partial_{\nu^{I}} \Theta\left[\begin{array}{l}\vec{\delta}_{1} \\ \vec{\delta}_{2}\end{array}\right](0 \mid \tau) \omega^{I}(z)$, and the Riemann Theta function is:

$$
\Theta\left[\begin{array}{l}
\vec{\delta}_{1} \\
\vec{\delta}_{2}
\end{array}\right](\vec{\nu} \mid \tau)=\sum_{\vec{n} \in \mathbb{Z}^{h}} e^{\pi i\left(n^{I}+\delta_{1}^{I}\right) \tau_{I J}\left(n^{J}+\delta_{1}^{J}\right)} e^{2 \pi i\left(n_{I}+\delta_{1 I}\right)\left(\nu^{I}+\delta_{2}^{I}\right)}
$$

The Riemann Theta function satisfies the following periodicity conditions:

$$
\Theta\left[\begin{array}{l}
\vec{\delta}_{1} \\
\vec{\delta}_{2}
\end{array}\right](\vec{\nu}+\vec{p}+\tau \vec{q} \mid \tau)=e^{-\pi q^{I} \tau_{I J} q^{J}-2 \pi i q_{I}\left(\nu^{I}+\delta_{2}^{I}\right)} e^{2 \pi i \delta_{1}^{I} p_{I}} \Theta\left[\begin{array}{l}
\vec{\delta}_{1} \\
\vec{\delta}_{2}
\end{array}\right](\vec{\nu} \mid \tau)
$$

with $(\vec{p}, \vec{q}) \in \mathbb{Z}^{2 h}$.

\section{Scalar Green function on genus $h$ Riemann surface}

In this appendix we discuss how the function $\mathcal{G}_{h}(z, w)$ introduced in eq. (2.10) is related to the scalar Green function on a genus $h$ Riemann surface.

\footnotetext{
${ }^{7}$ In ref. [86] the argument of the Theta-function is defined without the factor $1 / 2 \pi i$ in front of the integral, i.e. $\Theta\left[\left[_{\vec{\delta}_{2}}^{\vec{\delta}_{1}}\right]\left(\int_{z}^{w} \vec{\omega} \mid \tau\right)\right.$. However there also the abelian differentials are normalized without that factor, i.e. $\oint_{\left(a_{I} ; b_{I}\right)} \omega^{J}=\left(\delta_{I J} ; \tau_{I J}\right)$. The factor $2 \pi i$ in eq. (B.25) compensates these different normalizations.
} 
In eq. (2.90) of ref. [68] the regularized Green function is defined through the following relation:

$$
G_{r}\left(z_{i}, z_{j}\right)=G\left(z_{i}, z_{j}\right)-\frac{1}{2} G_{R}\left(z_{i}, z_{i}\right)-\frac{1}{2} G_{R}\left(z_{j}, z_{j}\right)=-\frac{1}{2} \log \left[\rho\left(z_{i}\right) \rho\left(z_{j}\right)\right]-\log F\left(z_{i}, z_{j}\right)
$$

where the Green function is defined as follows

$$
G\left(z_{i}, z_{j}\right)=\left\langle x\left(z_{i}\right) x\left(z_{j}\right)\right\rangle
$$

and $G_{R}$ provides the regularization at coincident points $z_{i}=z_{j}$ (see ref. [68] for explicit expressions). The metric in the conformal gauge has been chosen to be

$$
d s^{2}=\rho(z) d z d \bar{z}
$$

and the function $F$, defined by

$$
\log F\left(z_{i}, z_{j}\right)=\log \left|E\left(z_{i}, z_{j}\right)\right|^{2}+\operatorname{Re}\left(\int_{z_{j}}^{z_{i}} \omega_{I}\right)(2 \pi \operatorname{Im} \tau)_{I J}^{-1} \operatorname{Re}\left(\int_{z_{i}}^{z_{j}} \omega_{J}\right)
$$

was introduced in eqs. (2.90) and (2.91) of ref. [68], with $E$ being the prime form, $\omega_{I}$ the abelian differentials, and $\tau_{I J}$ the period matrix (see appendix B). ${ }^{8}$

If the conformal factors in eq. (C.1) are related to the choice of coordinates $V_{i}(z)$, entering in eq. (2.10), by

$$
\frac{1}{\rho\left(z_{i}\right)}=\left|V_{i}^{\prime}(0)\right|^{2} ; \quad \frac{1}{\rho\left(z_{j}\right)}=\left|V_{j}^{\prime}(0)\right|^{2}
$$

we see that eq. (C.1) implies that

$$
G_{r}(z, w)=-\mathcal{G}_{h}(z, w)
$$

In the following subsection we will argue that this coordinate choice can be consistently made. In a subsequent subsection we discuss some properties of $G_{r}$.

\section{C.1 Choice of coordinates}

The identification in eq. (C.6) is found through the relation in eq. (C.5) between $\rho(z)$ and $V_{i}^{\prime}(0)$. This choice of coordinates can be made if both sides of the relation transform in the same way under conformal transformations, which we will now check.

A coordinate system around a puncture is defined by the function:

$$
w_{i}=V_{i}^{-1}(z) \longrightarrow z=V_{i}\left(w_{i}\right)
$$

where $z$ is a global coordinate that gives $z_{i}$ for $w_{i}=0$ :

$$
z_{i}=V_{i}(0)
$$

\footnotetext{
${ }^{8}$ In ref. [68] the abelian differentials are normalized as $\int_{a_{J}} \omega_{K}=\delta_{J K}, \int_{b_{J}} \omega_{K}=\tau_{J K}$ while in our case they are normalized as (see appendix B): $\int_{a_{J}} \omega_{K}=2 \pi i \delta_{J K} ; \quad \int_{b_{J}} \omega_{K}=2 \pi i \tau_{J K}$.
} 
If we use another global parameter $\tilde{z}$ then we have the following relation

$$
w_{i}=\tilde{V}_{i}^{-1}(\tilde{z}) \longrightarrow \tilde{z}=\tilde{V}_{i}\left(w_{i}\right) ; \quad \tilde{z}_{i}=\tilde{V}_{i}(0)
$$

Since $w_{i}$ is the same in the two equations (C.7) and (C.9) we get:

$$
\frac{\partial \tilde{z}}{\partial z}\left(w_{i}=0\right)=\left.\frac{\partial \tilde{V}\left(w_{i}\right)}{\partial V_{i}\left(w_{i}\right)}\right|_{w_{i}=0}=\frac{\tilde{V}_{i}^{\prime}(0)}{V_{i}^{\prime}(0)} \Longrightarrow \tilde{V}_{i}^{\prime}(0)=\left.\frac{\partial \tilde{z}}{\partial z}\right|_{w_{i}=0} V_{i}^{\prime}(0)
$$

and hence $V_{i}^{\prime}(0)$ transforms as

$$
\tilde{V}_{i}^{\prime}(0) \tilde{\bar{V}}_{i}^{\prime}(0)=\left(\frac{\partial \tilde{z}}{\partial z} \frac{\partial \tilde{\bar{z}}}{\partial \bar{z}}\right)_{w_{i}=0} V_{i}^{\prime}(0) \bar{V}_{i}^{\prime}(0)
$$

Next we consider $\rho(z)$. Under an arbitrary reparametrization the metric transforms as follows:

$$
g_{\mu \nu}(x) \rightarrow \tilde{g}_{\mu \nu}(\tilde{x})=\frac{\partial x^{\rho}}{\partial \tilde{x}^{\mu}} \frac{\partial x^{\sigma}}{\partial \tilde{x}^{\nu}} g_{\mu \nu}(x)
$$

In the conformal gauge the previous relation becomes

$$
\rho(z) \rightarrow \tilde{\rho}(\tilde{z})=\frac{\partial z}{\partial \tilde{z}} \frac{\partial \bar{z}}{\partial \overline{\tilde{z}}} \rho(z)
$$

In particular, we get

$$
\rho\left(z_{i}\right) \rightarrow \tilde{\rho}\left(\tilde{z}_{i}\right)=\left(\frac{\partial z}{\partial \tilde{z}} \frac{\partial \bar{z}}{\partial \overline{\tilde{z}}}\right)_{w_{i}=0} \rho\left(z_{i}\right)
$$

Comparing eqs. (C.14) and (C.11), we see that they are consistent with the identification in eq. (C.5). In other words the quantity:

$$
\tilde{\rho}\left(\tilde{z}_{i}\right)\left|\tilde{V}_{i}^{\prime}(0)\right|^{2}=\rho\left(z_{i}\right)\left|V_{i}^{\prime}(0)\right|^{2}
$$

is invariant under the conformal coordinate transformations.

\section{C.2 Properties of the regularized Green function}

The regularized Green function, $G_{r}(z, w)$, satisfies the following equation:

$$
\partial_{z} \partial_{\bar{z}} G_{r}(z, w)=-\pi \delta^{(2)}(z-w)+\frac{2 \pi g_{z \bar{z}}}{\int d^{2} z \sqrt{g}} .
$$

In the conformal gauge, $d s^{2}=\rho d z d \bar{z}$, we have that

$$
g_{z \bar{z}}=\rho / 2 \quad \text { and } \quad \sqrt{g}=\rho / 2
$$

It follows that

$$
\int \partial_{z} \partial_{\bar{z}} G_{r}(z, w) d^{2} z=0 .
$$

because with our conventions for $d^{2} z$ we get that $\int d^{2} z \delta^{(2)}(z-w)=2$. 
It is easy to verify that $G_{r}$ is invariant under transport along the homology cycles of the Riemann surface. As an example we outline the proof in the case of the $b_{I}$ cycles. With the help of eqs. (B.12), (B.19), (B.24) and (C.13) we get:

$$
\begin{aligned}
-G_{r}\left(S_{I}\left(z_{1}\right), z_{2}\right) & =\log F\left(S_{I}\left(z_{1}\right), z_{2}\right)+\frac{1}{2} \log \left[\rho\left(S_{I}\left(z_{1}\right), S_{I}\left(\bar{z}_{1}\right)\right) \rho\left(z_{2}, \bar{z}_{2}\right)\right] \\
& =\log F\left(z_{1}, z_{2}\right)-\log \left|c_{I} z+d_{I}\right|^{2}+\frac{1}{2} \log \left[\rho\left(z_{1}, \bar{z}_{1}\right) \rho\left(z_{2}, \bar{z}_{2}\right)\right]+\log \left|c_{I} z+d_{I}\right|^{2} \\
& =-G_{r}\left(z_{1}, z_{2}\right)
\end{aligned}
$$

The $F$-function defined in eq. (C.4), and related to $G_{r}$ through eq. (C.1), satisfies according to ref. [68] the following equation:

$$
\partial_{z} \partial_{\bar{z}} \log F(z, w)=\pi \delta^{(2)}(z-w)-\frac{1}{4 \pi} \omega_{I}(z)(\operatorname{Im} \tau)_{I J}^{-1} \bar{\omega}_{J}(\bar{z})
$$

On the other hand, from eq. (C.1) and (C.16) we get

$$
\partial_{z} \partial_{\bar{z}} \log F(z, w)=\pi \delta^{(2)}(z-w)-\frac{2 \pi \rho(z)}{\int d^{2} z \rho(z)}-\frac{1}{2} \partial_{z} \partial_{\bar{z}} \log \rho(z)
$$

Equating (C.20) and (C.21) we thus find the relation

$$
\frac{1}{4 \pi} \omega_{I}(z)(\operatorname{Im} \tau)_{I J}^{-1} \bar{\omega}_{J}(\bar{z})=\frac{2 \pi \rho(z)}{\int d^{2} z \rho(z)}+\frac{1}{2} \partial_{z} \partial_{\bar{z}} \log \rho(z)
$$

We would like here to check this nontrivial relation. We can explicitly prove the integral version of this identity. In a two-dimensional euclidean conformally flat space-time with metric

$$
g_{a b}=\left(\begin{array}{cc}
0 & \frac{\rho}{2} \\
\frac{\rho}{2} & 0
\end{array}\right)=\frac{\rho}{2}\left(\begin{array}{cc}
0 & 1 \\
1 & 0
\end{array}\right) ; \quad g^{a b}=\frac{2}{\rho}\left(\begin{array}{cc}
0 & 1 \\
1 & 0
\end{array}\right)
$$

the curvature scalar is related to $\rho$ as follows

$$
R=-\frac{1}{\rho} \partial_{\mu} \partial^{\mu} \log \rho=-\frac{4}{\rho} \partial_{z} \partial_{\bar{z}} \log \rho
$$

where

$$
z=z_{1}+i z_{2} ; \quad \bar{z}=z_{1}-i z_{2} ; \quad \partial_{z}=\frac{1}{2}\left(\partial_{z_{1}}-i \partial_{z_{2}}\right) ; \quad \partial_{\bar{z}}=\frac{1}{2}\left(\partial_{z_{1}}+i \partial_{z_{2}}\right)
$$

Now, integrating eq. (C.22) over $d^{2} z$ and using the relation in eq. (3.5),

$$
\int d^{2} z \omega_{I}(z) \bar{\omega}_{J}(\bar{z})=2(2 \pi)^{2}(\operatorname{Im} \tau)_{I J}
$$

we get

$$
2 \pi h=2 \pi-\frac{1}{8} \int d^{2} z \rho R
$$


The Gauss-Bonnet theorem for a compact Riemann surface without boundary states that

$$
\frac{1}{4 \pi} \int \sqrt{g} R=2(1-h)
$$

and since $\sqrt{g}=\rho / 2$ we see that eq. (C.27) is indeed satisfied.

We can furthermore check eq. (C.22) explicitly at one loop. At one-loop we have

$$
\omega(z)=\frac{1}{z} ; \quad \bar{\omega}(\bar{z})=\frac{1}{\bar{z}} ; \quad \operatorname{Im} \tau \equiv \tau_{2} ; \quad R=0
$$

Then, eq. (C.22), using eq. (C.24), becomes

$$
\frac{1}{4 \pi|z|^{2} \tau_{2}}=\frac{2 \pi \rho(z)}{\int d^{2} z \rho(z)}
$$

Assuming that, at one-loop, $\rho(z)$ is equal to (which we prove in the end)

$$
\rho(z)=\frac{1}{(2 \pi)^{2}|z|^{2}}=\frac{1}{(2 \pi)^{2}} \omega(z) \bar{\omega}(\bar{z})
$$

we can compute the volume integral in the denominator of the r.h.s.

$$
\begin{aligned}
\int d^{2} z \rho(z) & =\frac{1}{(2 \pi)^{2}} \int \frac{d^{2} z}{|z|^{2}}=\frac{1}{(2 \pi)^{2}} \int \frac{d z \wedge d \bar{z}}{i z} \partial_{\bar{z}} \log |z|^{2} \\
& =\frac{1}{(2 \pi)^{2}}\left(\oint_{|z|=1} \frac{d z}{i z} \log |z|^{2}-\oint_{|z|=|\kappa|} \frac{d z}{i z} \log |z|^{2}\right)=-\frac{2 \pi}{(2 \pi)^{2}} \log |\kappa|^{2} \\
& =2 \tau_{2}
\end{aligned}
$$

where we have used the relation:

$$
|\kappa|^{2}=\mathrm{e}^{-4 \pi \tau_{2}}
$$

and the fact that the integral is performed in the region of the $z$-plane contained between the circle of radius 1 and that of radius $|\kappa|$. Consistently, the result of eq. (C.32) follows also immediately from eq. (C.26) and eq. (C.31). Eqs. (C.31) and (C.32) imply that eq. (C.30) is satisfied.

Finally, comparing eqs. (C.5) and (C.31), at one-loop, we get [88]:

$$
V_{i}^{\prime}(0)=2 \pi z_{i}
$$

The same result can be obtained by observing that the one-loop world-sheet in the closed string is a torus which is a flat manifold. Parametrizing the manifold with real coordinates $(\tau, \sigma)$, the metric will be given by $d s^{2}=\mathrm{d} \tau^{2}+d \sigma^{2}$. By rewriting the metric in terms of the complex coordinates $z=e^{2 \pi(\tau-i \sigma)}$ and $\bar{z}=e^{2 \pi(\tau+i \sigma)}$, one gets:

$$
d s^{2}=\mathrm{d} \tau^{2}+d \sigma^{2}=\frac{d z d \bar{z}}{(2 \pi)^{2}|z|^{2}}
$$

It is then immediately seen that the 1-loop conformal factor of the metric is exactly the one written in eq. (C.31) 


\section{Derivation of the $N$-Reggeon vertex}

In this appendix we derive the $N$-Reggeon Vertex for the closed bosonic string given in eq. (2.1) starting from eq. (3.24) of ref. [59]. We start by noticing that the terms in the first line of eq. (3.23) of the reference cancel with the terms in the denominator in the log of the second line of eq. (3.24) with $i \neq j$. This happens for both the holomorphic and anti-holomorphic variables. This means that the vertex in eq. (3.24) of ref. [59] can be written as follows:

$$
\begin{aligned}
& V_{N}= C_{h} N_{0}^{N} \int d V\langle\Omega| \prod_{i=1}^{N}\left[\left|V_{i}^{\prime}(0)\right|^{\frac{\alpha^{\prime} p_{i}^{2}}{2}}\right] \exp \left[\left.\frac{1}{2} \sum_{i=1}^{N} \sum_{n=1}^{\infty} \frac{\alpha_{n}^{(i)}}{n !} \alpha_{0}^{(i)} \frac{\partial^{n}}{\partial z^{n}} \log V^{\prime}(z)\right|_{z=0}\right] \\
& \times \exp \left[\left.\frac{1}{2} \sum_{i=1}^{N} \sum_{n=1}^{\infty} \frac{\bar{\alpha}_{n}^{(i)}}{n !} \alpha_{0}^{(i)} \frac{\partial^{n}}{\partial \bar{z}^{n}} \log \bar{V}^{\prime}(\bar{z})\right|_{\bar{z}=0}\right] \\
& \times {\left[\left.\frac{1}{2} \sum_{i \neq j} \sum_{n, m=0}^{\infty} \frac{\alpha_{n}^{(i)}}{n !} \partial_{z}^{n} \partial_{y}^{m} \log E\left(V_{i}(z), V_{j}(y)\right)\right|_{z=y=0} \frac{\alpha_{m}^{(j)}}{m !}\right] } \\
& \times \exp \left[\left.\frac{1}{2} \sum_{i \neq j} \sum_{n, m=0}^{\infty} \frac{\bar{\alpha}_{n}^{(i)}}{n !} \partial_{\bar{z}}^{n} \partial_{\bar{y}}^{m} \log E\left(\bar{V}_{i}(\bar{z}), \bar{V}_{j}(\bar{y})\right)\right|_{z=y=0} \frac{\bar{\alpha}_{m}^{(j)}}{m !}\right] \\
& \times \exp \left[\left.\frac{1}{2} \sum_{i=1}^{N} \sum_{n, m=0}^{\infty} \alpha_{n}^{(i)} \frac{1}{n ! m !} \partial_{z}^{n} \partial_{y}^{m} \log \frac{E\left(V_{i}(z), V_{i}(y)\right)}{V_{i}(z)-V_{i}(y)}\right|_{z=y=0} \alpha_{m}^{(i)}\right] \\
& \times \exp \left[\left.\frac{1}{2} \sum_{i=1}^{N} \sum_{n, m=0}^{\infty} \bar{\alpha}_{n}^{(i)} \frac{1}{n ! m !} \partial_{\bar{z}}^{n} \partial_{\bar{y}}^{m} \log \frac{E\left(\bar{V}_{i}(\bar{z}), \bar{V}_{i}(\bar{y})\right)}{\bar{V}_{i}(\bar{z})-\bar{V}_{i}(\bar{y})}\right|_{\bar{z}=\bar{y}=0} \bar{\alpha}_{m}^{(i)}\right] \\
& \times \exp \left[\sum_{i, j=1}^{N} \sum_{n=0}^{\infty}\left(\frac{\alpha_{n}^{(i)}}{n !} \partial_{z}^{n}+\frac{\bar{\alpha}_{n}^{(i)}}{n !} \partial_{\bar{z}}^{n}\right) \operatorname{Re}\left(\int_{z_{0}}^{V_{i}(z)} \omega_{I}\right)(2 \pi \operatorname{Im} \tau)_{I J}^{-1}\right. \\
&\left.\times \sum_{m=0}^{\infty}\left(\frac{\alpha_{m}^{(j)}}{n !} \partial_{z}^{m}+\frac{\bar{\alpha}_{m}^{(i)}}{m !} \partial_{\bar{z}}^{m}\right) \operatorname{Re}\left(\int_{z_{0}}^{V_{j}(z)} \omega_{J}\right)\right]
\end{aligned}
$$

where we have also integrated over the momenta circulating in the $h$ loops. The final vertex is written in eq. (2.1) where, for reasons that will become clear later, using momentum conservation, we have put the factor in front containing $V_{i}^{\prime}(0)$ together with the primeform. This has been done by rewriting it as follows:

$$
\prod_{i=1}^{N}\left[\left(V_{i}^{\prime}(0)\right)^{\frac{\alpha^{\prime} p_{i}^{2}}{2}}\right]=\exp \left[-\frac{\alpha^{\prime}}{2} \sum_{i<j=1}^{N} p_{i} p_{j} \log \sqrt{V_{i}^{\prime}(0) V_{j}^{\prime}(0)}\right]
$$

Re In the vertex in eq. (2.1) we have also eliminated the dependence on the arbitrary point $z_{0}$. This is shown in the following. 
Let us consider the part containing the momentum given by

$$
\begin{aligned}
\sum_{i, j} p_{i} p_{j} \operatorname{Re} & \left.\left(\int_{z_{0}}^{V_{i}(z)} \omega_{I}\right)(2 \pi \operatorname{Im} \tau)_{I J}^{-1} \operatorname{Re}\left(\int_{z_{0}}^{V_{j}(y)} \omega_{J}\right)\right|_{z=y=0} \\
= & \left.\sum_{i \neq j} p_{i} p_{j} \operatorname{Re}\left(\int_{z_{0}}^{V_{j}(y)}+\int_{V_{j}(y)}^{V_{i}(z)}\right) \omega_{I}(2 \pi \operatorname{Im} \tau)_{I J}^{-1} \operatorname{Re} \int_{z_{0}}^{V_{j}(y)} \omega_{J}\right|_{z=y=0} \\
& +\left.\sum_{i} p_{i}^{2} \operatorname{Re}\left(\int_{z_{0}}^{V_{i}(z)} \omega_{I}\right)(2 \pi \operatorname{Im} \tau)_{I J}^{-1} \operatorname{Re}\left(\int_{z_{0}}^{V_{i}(z)} \omega_{J}\right)\right|_{z=y=0} \\
= & \left.\sum_{i \neq j} p_{i} p_{j} \operatorname{Re}\left(\int_{V_{j}(y)}^{V_{i}(z)} \omega_{I}\right)(2 \pi \operatorname{Im} \tau)_{I J}^{-1} \operatorname{Re}\left(\int_{z_{0}}^{V_{j}(y)} \omega_{J}\right)\right|_{z=y=0} \\
& -\left.\sum_{j} p_{j}^{2} \operatorname{Re}\left(\int_{z_{0}}^{V_{j}(y)} \omega_{I}\right)(2 \pi \operatorname{Im} \tau)_{I J}^{-1} \operatorname{Re}\left(\int_{z_{0}}^{V_{j}(y)} \omega_{J}\right)\right|_{z=y=0} \\
& +\left.\sum_{i} p_{i}^{2} \operatorname{Re}\left(\int_{z_{0}}^{V_{i}(z)} \omega_{I}\right)(2 \pi \operatorname{Im} \tau)_{I J}^{-1} \operatorname{Re}\left(\int_{z_{0}}^{V_{i}(z)} \omega_{J}\right)\right|_{z=y=0} \\
= & \left.\sum_{i \neq j} p_{i} p_{j} \operatorname{Re}\left(\int_{V_{j}(y)}^{V_{i}(z)} \omega_{I}\right)(2 \pi \operatorname{Im} \tau)_{I J}^{-1} \operatorname{Re}\left(\int_{z_{0}}^{V_{j}(y)} \omega_{J}\right)\right|_{z=y=0}
\end{aligned}
$$

In conclusion, we get

$$
\begin{aligned}
\left.\sum_{i, j} p_{i} p_{j} \operatorname{Re}\left(\int_{z_{0}}^{V_{i}(z)} \omega_{I}\right)(2 \pi \operatorname{Im} \tau)_{I J}^{-1} \operatorname{Re}\left(\int_{z_{0}}^{V_{j}(y)} \omega_{J}\right)\right|_{z=y=0} \\
=\left.\sum_{i, j} p_{i} p_{j} \operatorname{Re}\left(\int_{V_{j}(y)}^{V_{i}(z)} \omega_{I}\right)(2 \pi \operatorname{Im} \tau)_{I J}^{-1} \operatorname{Re}\left(\int_{z_{0}}^{V_{j}(y)} \omega_{J}\right)\right|_{z=y=0}
\end{aligned}
$$

where in the last step we have added the terms $i=j$ because they do not give any contribution. In order to see that the quantity on the 1.h.s. of the previous equation is independent of $z_{0}$, we rewrite it as follows:

$$
\begin{gathered}
\left.\sum_{i, j} p_{i} p_{j} \operatorname{Re}\left(\int_{z_{0}}^{V_{i}(z)} \omega_{I}\right)(2 \pi \operatorname{Im} \tau)_{I J}^{-1} \operatorname{Re}\left(\int_{z_{0}}^{V_{j}(y)} \omega_{J}\right)\right|_{z=y=0} \\
=\left.\sum_{i, j} p_{i} p_{j} \operatorname{Re}\left[\left(\int_{z_{0}}^{V_{j}(y)}+\int_{V_{j}(y)}^{V_{i}(z)}\right) \omega_{I}\right](2 \pi \operatorname{Im} \tau)_{I J}^{-1} \operatorname{Re}\left[\left(\int_{z_{0}}^{V_{i}(z)}+\int_{V_{i}(z)}^{V_{j}(y)}\right) \omega_{J}\right]\right|_{z=y=0}
\end{gathered}
$$


that implies

$$
\begin{aligned}
\left.\sum_{i, j} p_{i} p_{j} \operatorname{Re}\left(\int_{V_{j}(y)}^{V_{i}(z)}\right) \omega_{I}(2 \pi \operatorname{Im} \tau)_{I J}^{-1} \operatorname{Re}\left(\int_{V_{i}(z)}^{V_{j}(y)}\right) \omega_{J}\right|_{z=y=0} \\
=-\left.\sum_{i, j} p_{i} p_{j} \operatorname{Re}\left(\int_{z_{0}}^{V_{j}(y)}\right) \omega_{I}(2 \pi \operatorname{Im} \tau)_{I J}^{-1} \operatorname{Re}\left(\int_{V_{i}(z)}^{V_{j}(y)}\right) \omega_{J}\right|_{z=y=0} \\
\quad-\left.\sum_{i, j} p_{i} p_{j} \operatorname{Re}\left(\int_{V_{j}(y)}^{V_{i}(z)}\right) \omega_{I}(2 \pi \operatorname{Im} \tau)_{I J}^{-1} \operatorname{Re}\left(\int_{z_{0}}^{V_{i}(z)}\right) \omega_{J}\right|_{z=y=0} \\
=-\left.2 \sum_{i, j} p_{i} p_{j} \operatorname{Re}\left(\int_{V_{i}(z)}^{V_{j}(y)}\right) \omega_{I}(2 \pi \operatorname{Im} \tau)_{I J}^{-1} \operatorname{Re}\left(\int_{z_{0}}^{V_{j}(y)}\right) \omega_{J}\right|_{z=y=0}
\end{aligned}
$$

where we have used the fact that the terms in the second and third lines are equal as one can see by exchanging the indices $i$ with $j$ in one of them. Comparing the previous equation with eq. (D.4) we get the final result

$$
\begin{gathered}
\left.\sum_{i, j} p_{i} p_{j} \operatorname{Re}\left(\int_{z_{0}}^{V_{i}(z)} \omega_{I}\right)(2 \pi \operatorname{Im} \tau)_{I J}^{-1} \operatorname{Re}\left(\int_{z_{0}}^{V_{j}(y)} \omega_{J}\right)\right|_{z=y=0} \\
=\left.\frac{1}{2} \sum_{i, j} p_{i} p_{j} \operatorname{Re}\left(\int_{V_{j}(y)}^{V_{i}(z)} \omega_{I}\right)(2 \pi \operatorname{Im} \tau)_{I J}^{-1} \operatorname{Re}\left(\int_{V_{i}(z)}^{V_{j}(y)} \omega_{J}\right)\right|_{z=y=0}
\end{gathered}
$$

that shows independence on $z_{0}$ for the terms with two momenta. The terms involving only the oscillators do not have any dependence on $z_{0}$. The terms with an oscillator and a momentum do not depend on $z_{0}$ because of momentum conservation. Therefore we can eliminate $z_{0}$ everywhere as in eq. (D.7). This ends the derivation of eq. (2.1).

\section{E Calculation of $S_{1}$}

Let us for brevity make some definitions and recall some identities,

$$
\begin{aligned}
\partial_{\bar{z}_{1}} \partial_{z_{1}} \mathcal{G}\left(z_{1}, z_{2}\right) & =\pi \delta^{(2)}\left(z_{1}-z_{2}\right)+\mathcal{T}\left(z_{1}\right) \\
\int_{\Sigma_{h}} d^{2} z \partial_{\bar{z}} \partial_{z} \mathcal{G}(z, w) & =0 \quad \Leftrightarrow \quad \int_{\Sigma_{h}} d^{2} z \mathcal{T}(z)=-2 \pi \\
e^{\frac{\alpha^{\prime}}{2} k_{i} q \mathcal{G}\left(z_{i}, z_{i}\right)} \propto\left|E\left(z_{i}, z_{i}\right)\right|^{\alpha^{\prime} k_{i} q} & =0
\end{aligned}
$$

where $\mathcal{G}$ is the quantity defined in eq. (2.10), however, in this section we suppress the index $h$ for brevity. These relations apply for any number of loops $h \geq 1$, and are sufficient to determine the soft behavior of $S_{1}$ defined in eq. (3.3) through order $q$ in terms of $\mathcal{G}$ only, which will here be shown. We repeat the definition of $S_{1}$ and decompose the integrand in 
three parts:

$$
\begin{aligned}
S_{1}= & \int \mathrm{d}^{2} z\left[1+\sum_{j \neq i}^{n} \frac{\alpha^{\prime}}{2} k_{j} q \mathcal{G}_{h}\left(z_{i}, z\right)+\frac{1}{2}\left(\frac{\alpha^{\prime}}{2}\right)^{2} \sum_{j, l \neq i}^{n}\left(k_{j} q\right)\left(k_{l} q\right) \mathcal{G}_{h}\left(z_{j}, z\right) \mathcal{G}_{h}\left(z_{l}, z\right)\right] \\
& \times \frac{\alpha^{\prime}}{2} \sum_{i, j=1}^{n}\left(k_{i} \epsilon\right)\left(k_{j} \bar{\epsilon}\right) \partial_{z} \mathcal{G}_{h}\left(z_{i}, z\right) \partial_{\bar{z}} \mathcal{G}_{h}\left(z_{j}, z\right) e^{\frac{\alpha^{\prime}}{2}} k_{i} q \mathcal{G}_{h}\left(z_{i}, z\right)+\mathcal{O}\left(q^{2}\right) \\
= & I_{0}+\left(\frac{\alpha^{\prime}}{2}\right) I_{1}+\frac{1}{2}\left(\frac{\alpha^{\prime}}{2}\right)^{2} I_{2}+\mathcal{O}\left(q^{2}\right)
\end{aligned}
$$

In the following subsections we will make substantial use of integration by parts, neglecting boundary (total derivative) terms, to compute the three terms above. Remarkably, the three terms can be fully computed by only using the above properties of Green's function; i.e. without knowing its explicit form, in the case where the soft state is symmetrically polarized. We summarize here the results of the calculation:

$$
\begin{aligned}
I_{0}= & 2 \pi \sum_{i} \frac{\left(k_{i} \epsilon_{q}\right)\left(k_{i} \bar{\epsilon}_{q}\right)}{k_{i} q}-2 \pi \frac{\alpha^{\prime}}{2} \sum_{i \neq j}\left(k_{i} \epsilon_{q}\right)\left(k_{j} \bar{\epsilon}_{q}\right) \mathcal{G}\left(z_{j}, z_{i}\right)\left[1+\frac{\alpha^{\prime}}{4} k_{i} q \mathcal{G}\left(z_{j}, z_{i}\right)\right] \\
I_{1}= & 2 \pi \epsilon_{q \mu \nu}^{S} \sum_{i \neq j} \frac{k_{i}^{\mu} k_{i}^{\nu}}{k_{i} q}\left(k_{j} q\right) \mathcal{G}\left(z_{j}, z_{i}\right)+\frac{2 \pi \alpha^{\prime}}{4} \epsilon_{q \mu \nu}^{S} \sum_{i \neq j} k_{i}^{\mu} k_{j}^{\nu}\left(k_{j} q\right) \mathcal{G}^{2}\left(z_{i}, z_{j}\right) \\
& +\frac{2 \pi \alpha^{\prime}}{4} \epsilon_{q \mu \nu}^{S} \sum_{i \neq j, l}\left[k_{j}^{\mu} k_{l}^{\nu}\left(k_{i} q\right)-k_{i}^{\mu} k_{l}^{\nu}\left(k_{j} q\right)-k_{i}^{\mu} k_{j}^{\nu}\left(k_{l} q\right)\right] \mathcal{G}\left(z_{i}, z_{j}\right) \mathcal{G}\left(z_{i}, z_{l}\right) \\
I_{2}= & 2 \pi \sum_{i=1}\left(k_{i} \epsilon_{q}\right)\left(k_{i} \bar{\epsilon}_{q}\right) \sum_{j, l \neq i} \frac{\left(k_{j} q\right)\left(k_{l} q\right)}{q k_{i}} \mathcal{G}\left(z_{i}, z_{j}\right) \mathcal{G}\left(z_{i}, z_{l}\right)
\end{aligned}
$$

Only in computing a particular integral in $I_{1}$ was it necessary to impose polarization symmetry on $\epsilon_{q \mu \nu}=\epsilon_{q \mu} \bar{\epsilon}_{q \nu}$, and this was in fact not necessary for the leading order $\mathcal{O}\left(q^{0}\right)$ term in $I_{1}$. Thus through order $q^{0}$ the result is valid for any of the three physical states of the massless closed string; the graviton, the dilaton and the Kalb-Ramond B-field.

\section{E.1 Calculation of $I_{0}$}

The integral $I_{0}$ can be written as:

$$
I_{0}=\sum_{i, j} \frac{\left(k_{i} \epsilon_{q}\right)\left(k_{j} \bar{\epsilon}_{q}\right)}{k_{i} q} \int d^{2} z\left[\partial_{z}\left(\partial_{\bar{z}} \mathcal{G}\left(z, z_{j}\right) e^{\frac{\alpha^{\prime}}{2} k_{i} q \mathcal{G}\left(z, z_{i}\right)}\right)-\partial_{\bar{z}} \partial_{z} \mathcal{G}\left(z, z_{j}\right) e^{\frac{\alpha^{\prime}}{2} k_{i} q \mathcal{G}\left(z, z_{i}\right)}\right]
$$


The boundary term vanishes, and in the second term the definition in eq. (E.1) can be inserted to get

$$
\begin{aligned}
I_{0} & =-\sum_{i, j} \frac{\left(k_{i} \epsilon_{q}\right)\left(k_{j} \bar{\epsilon}_{q}\right)}{k_{i} q} \int d^{2} z\left[\pi \delta^{2}\left(z-z_{j}\right)+\mathcal{T}(z)\right] e^{\frac{\alpha^{\prime}}{2} k_{i} q \mathcal{G}\left(z, z_{i}\right)} \\
& =-2 \pi \sum_{i, j} \frac{\left(k_{i} \epsilon_{q}\right)\left(k_{j} \bar{\epsilon}_{q}\right)}{k_{i} q} e^{\frac{\alpha^{\prime}}{2} k_{i} q \mathcal{G}\left(z_{j}, z_{i}\right)}-\sum_{i} \frac{\left(k_{i} \epsilon_{q}\right)\left(-q \bar{\epsilon}_{q}\right)}{k_{i} q} \int d^{2} z \mathcal{T}(z) e^{\frac{\alpha^{\prime}}{2} k_{i} q \mathcal{G}\left(z, z_{i}\right)} \\
& =-2 \pi \sum_{i \neq j} \frac{\left(k_{i} \epsilon_{q}\right)\left(k_{j} \bar{\epsilon}_{q}\right)}{k_{i} q} e^{\frac{\alpha^{\prime}}{2} k_{i} q \mathcal{G}\left(z_{j}, z_{i}\right)}
\end{aligned}
$$

To arrive at the last equality we used identity (E.3) as well as $\sum_{j}\left(k_{j} \bar{\epsilon}_{q}\right)=-\left(q \bar{\epsilon}_{q}\right)=0$.

By expanding the previous expression in the soft-momentum, the first term of the expansion is the Weinberg soft theorem, i.e.:

$$
\sum_{i \neq j} \frac{\left(k_{i} \epsilon_{q}\right)\left(k_{j} \bar{\epsilon}_{q}\right)}{k_{i} q}=-\sum_{i} \frac{\left(k_{i} \epsilon_{q}\right)\left(\left(k_{i}+q\right) \bar{\epsilon}_{q}\right)}{k_{i} q}=-\sum_{i} \frac{\left(k_{i} \epsilon_{q}\right)\left(k_{i} \bar{\epsilon}_{q}\right)}{k_{i} q}
$$

Thus, in conclusion:

$$
I_{0}=2 \pi \sum_{i} \frac{\left(k_{i} \epsilon_{q}\right)\left(k_{i} \bar{\epsilon}_{q}\right)}{k_{i} q}-2 \pi \frac{\alpha^{\prime}}{2} \sum_{i \neq j}\left(k_{i} \epsilon_{q}\right)\left(k_{j} \bar{\epsilon}_{q}\right) \mathcal{G}\left(z_{j}, z_{i}\right)\left[1+\frac{\alpha^{\prime}}{4} k_{i} q \mathcal{G}\left(z_{j}, z_{i}\right)\right]+O\left(q^{2}\right)
$$

This result is formally identically to the tree-level result in ref. [66], with the difference being in the Green function.

\section{E.2 Calculation of $I_{1}$}

The integral $I_{1}$ can be written as

$$
I_{1}=\sum_{i j} \frac{\left(k_{i} \epsilon_{q}\right)\left(k_{j} \bar{\epsilon}_{q}\right)}{k_{i} q} \sum_{l \neq i}\left(k_{l} q\right) \int d^{2} z \mathcal{G}\left(z, z_{l}\right) \partial_{\bar{z}} \mathcal{G}\left(z, z_{j}\right) \partial_{z} e^{\frac{\alpha^{\prime}}{2} k_{i} q \mathcal{G}\left(z, z_{i}\right)}
$$

and integration by parts gives:

$$
\begin{aligned}
I_{1}= & -\sum_{i j} \frac{\left(k_{i} \epsilon_{q}\right)\left(k_{j} \bar{\epsilon}_{q}\right)}{k_{i} q} \sum_{l \neq i}\left(k_{l} q\right) \int d^{2} z \partial_{\bar{z}} \mathcal{G}\left(z, z_{j}\right) \partial_{z} \mathcal{G}\left(z, z_{l}\right) e^{\frac{\alpha^{\prime}}{2} k_{i} q \mathcal{G}\left(z, z_{i}\right)} \\
& -\sum_{i j} \frac{\left(k_{i} \epsilon_{q}\right)\left(k_{j} \bar{\epsilon}_{q}\right)}{k_{i} q} \sum_{l \neq i}\left(k_{l} q\right) \int d^{2} z \mathcal{G}\left(z, z_{l}\right) \partial_{\bar{z}} \partial_{z} \mathcal{G}\left(z, z_{j}\right) e^{\frac{\alpha^{\prime}}{2} k_{i} q \mathcal{G}\left(z, z_{i}\right)}
\end{aligned}
$$

The integral in the second line gives:

$$
I_{1}^{(2)}=-\sum_{i j} \frac{\left(k_{i} \epsilon_{q}\right)\left(k_{j} \bar{\epsilon}_{q}\right)}{k_{i} q} \sum_{l \neq i}\left(k_{l} q\right) \int d^{2} z \mathcal{G}\left(z, z_{l}\right)\left[\pi \delta^{2}\left(z-z_{j}\right)+\mathcal{T}(z)\right] e^{\frac{\alpha^{\prime}}{2} k_{i} q \mathcal{G}\left(z, z_{i}\right)}
$$

Notice that for the second term we can replace by momentum conservation $\sum_{j} k_{j} \bar{\epsilon}_{q}=$ $-q \epsilon_{q}=0$, and hence only the first term remains, which after expansion reads:

$$
I_{1}^{(2)}=-2 \pi \sum_{i \neq j} \frac{\left(k_{i} \epsilon_{q}\right)\left(k_{j} \bar{\epsilon}_{q}\right)}{k_{i} q} \sum_{l \neq i}\left(k_{l} q\right) \mathcal{G}\left(z_{j}, z_{l}\right)\left[1+\frac{\alpha^{\prime}}{2} k_{i} q \mathcal{G}\left(z_{j}, z_{i}\right)\right]+O\left(q^{2}\right)
$$


where we used that for $i=j$ the expression is zero, before expansion, due to eq. (E.3). For $l=j$ there is a divergence, but as we will see, it cancels against another term, coming from the first integral in eq. (E.13).

To calculate the first integral in eq. (E.13), we consider the cases $i=j$ and $i \neq j$ separately. The case $i=j$ can be written as:

$$
\begin{aligned}
\left.I_{1}^{(1)}\right|_{i=j} & =-\sum_{i} \frac{\left(k_{i} \epsilon_{q}\right)\left(k_{i} \bar{\epsilon}_{q}\right)}{k_{i} q} \sum_{l \neq i} \frac{\left(k_{l} q\right)}{\frac{\alpha^{\prime}}{2}\left(k_{i} q\right)} \int d^{2} z \partial_{z} \mathcal{G}\left(z, z_{l}\right) \partial_{\bar{z}} e^{\frac{\alpha^{\prime}}{2} k_{i} q \mathcal{G}\left(z, z_{i}\right)} \\
& =+\sum_{i} \frac{\left(k_{i} \epsilon_{q}\right)\left(k_{i} \bar{\epsilon}_{q}\right)}{k_{i} q} \sum_{l \neq i} \frac{\left(k_{l} q\right)}{\frac{\alpha^{\prime}}{2}\left(k_{i} q\right)} \int d^{2} z \partial_{z} \partial_{\bar{z}} \mathcal{G}\left(z, z_{l}\right) e^{\frac{\alpha^{\prime}}{2} k_{i} q \mathcal{G}\left(z, z_{i}\right)}
\end{aligned}
$$

Using eq. (E.2) and eq. (E.1) we get:

$$
\begin{aligned}
& \left.I_{1}^{(1)}\right|_{i=j}=2 \pi \sum_{i} \frac{\left(k_{i} \epsilon_{q}\right)\left(k_{i} \bar{\epsilon}_{q}\right)}{k_{i} q} \sum_{l \neq i} \frac{\left(k_{l} q\right)}{\frac{\alpha^{\prime}}{2}\left(k_{i} q\right)}\left[e^{\frac{\alpha^{\prime}}{2} k_{i} q \mathcal{G}\left(z_{l}, z_{i}\right)}-1\right] \\
& +\sum_{i} \frac{\left(k_{i} \epsilon_{q}\right)\left(k_{i} \bar{\epsilon}_{q}\right)}{k_{i} q} \sum_{l \neq i}\left(k_{l} q\right) \int d^{2} z \mathcal{T}(z) \mathcal{G}\left(z, z_{i}\right) \\
& +\frac{\alpha^{\prime}}{4} \sum_{i}\left(k_{i} \epsilon_{q}\right)\left(k_{i} \bar{\epsilon}_{q}\right) \sum_{l \neq i}\left(k_{l} q\right) \int d^{2} z \mathcal{T}(z) \mathcal{G}^{2}\left(z, z_{i}\right)+O\left(q^{2}\right) \\
& =2 \pi \sum_{i} \frac{\left(k_{i} \epsilon_{q}\right)\left(k_{i} \bar{\epsilon}_{q}\right)}{k_{i} q} \sum_{l \neq i}\left(k_{l} q\right) \mathcal{G}\left(z_{l}, z_{i}\right)+\frac{2 \pi \alpha^{\prime}}{4} \sum_{i}\left(k_{i} \epsilon_{q}\right)\left(k_{i} \bar{\epsilon}_{q}\right) \sum_{l \neq i}\left(k_{l} q\right) \mathcal{G}^{2}\left(z_{l}, z_{i}\right) \\
& -\sum_{i}\left(k_{i} \epsilon_{q}\right)\left(k_{i} \bar{\epsilon}_{q}\right) \int d^{2} z \mathcal{T}(z) \mathcal{G}\left(z, z_{i}\right) \\
& -\frac{\alpha^{\prime}}{4} \sum_{i}\left(k_{i} \epsilon_{q}\right)\left(k_{i} \bar{\epsilon}_{q}\right)\left(k_{i} q\right) \int d^{2} z \mathcal{T}(z) \mathcal{G}^{2}\left(z, z_{i}\right)+O\left(q^{2}\right)
\end{aligned}
$$

where we have used $\sum_{l \neq i} k_{l} q=-k_{i} q$ in the last two terms. We will see in a moment that the second-to-last (blue) line cancels out with another term.

In the case $i \neq j$ we make instead the following rewriting:

$$
\begin{aligned}
\left.I_{1}^{(1)}\right|_{i \neq j}= & -\sum_{i \neq j} \frac{\left(k_{i} \epsilon_{q}\right)\left(k_{j} \bar{\epsilon}_{q}\right)}{k_{i} q} \sum_{l \neq i}\left(k_{l} q\right) \int d^{2} z \partial_{\bar{z}}\left[\partial_{z} \mathcal{G}\left(z, z_{l}\right) \mathcal{G}\left(z, z_{j}\right)\right] e^{\frac{\alpha^{\prime}}{2} k_{i} q \mathcal{G}\left(z, z_{i}\right)} \\
& +\sum_{i \neq j} \frac{\left(k_{i} \epsilon_{q}\right)\left(k_{j} \bar{\epsilon}_{q}\right)}{k_{i} q} \sum_{l \neq i}\left(k_{l} q\right) \int d^{2} z \partial_{z} \partial_{\bar{z}} \mathcal{G}\left(z, z_{l}\right) \mathcal{G}\left(z, z_{j}\right) e^{\frac{\alpha^{\prime}}{2} k_{i} q \mathcal{G}\left(z, z_{i}\right)}
\end{aligned}
$$

We can expand the exponentials, since around the poles of the integrand, $z=z_{l}$ and $z=z_{j}$, the exponential is regular $(i \neq j, l)$. This gets rid of a total derivative in the first term, and 
by using eq. (E.1) in the second term one finds:

$$
\begin{aligned}
\left.I_{1}^{(1)}\right|_{i \neq j}= & -\frac{\alpha^{\prime}}{2} \sum_{i \neq j}\left(k_{i} \epsilon_{q}\right)\left(k_{j} \bar{\epsilon}_{q}\right) \sum_{l \neq i}\left(k_{l} q\right) \int d^{2} z \partial_{\bar{z}}\left[\partial_{z} \mathcal{G}\left(z, z_{l}\right) \mathcal{G}\left(z, z_{j}\right)\right] \mathcal{G}\left(z, z_{i}\right) \\
& +2 \pi \sum_{i \neq j} \frac{\left(k_{i} \epsilon_{q}\right)\left(k_{j} \bar{\epsilon}_{q}\right)}{k_{i} q} \sum_{l \neq i}\left(k_{l} q\right) \mathcal{G}\left(z_{l}, z_{j}\right)\left[1+\frac{\alpha^{\prime}}{2} k_{i} q \mathcal{G}\left(z_{l}, z_{i}\right)\right] \\
& -\sum_{i \neq j}\left(k_{i} \epsilon_{q}\right)\left(k_{j} \bar{\epsilon}_{q}\right) \int d^{2} z \mathcal{T}(z) \mathcal{G}\left(z, z_{j}\right)\left[1+\frac{\alpha^{\prime}}{2} k_{i} q \mathcal{G}\left(z, z_{i}\right)\right]+O\left(q^{2}\right)
\end{aligned}
$$

By summing the (red) second term with eq. (E.15) we get:

$$
\begin{aligned}
& -2 \pi \sum_{i \neq j} \frac{\left(k_{i} \epsilon_{q}\right)\left(k_{j} \bar{\epsilon}_{q}\right)}{k_{i} q} \sum_{l \neq i}\left(k_{l} q\right) \mathcal{G}\left(z_{j}, z_{l}\right)\left[1+\frac{\alpha^{\prime}}{2} k_{i} q \mathcal{G}\left(z_{j}, z_{i}\right)\right] \\
& +2 \pi \sum_{i \neq j} \frac{\left(k_{i} \epsilon_{q}\right)\left(k_{j} \bar{\epsilon}_{q}\right)}{k_{i} q} \sum_{l \neq i}\left(k_{l} q\right) \mathcal{G}\left(z_{l}, z_{j}\right)\left[1+\frac{\alpha^{\prime}}{2} k_{i} q \mathcal{G}\left(z_{l}, z_{i}\right)\right] \\
& \quad=\frac{2 \pi \alpha^{\prime}}{2} \sum_{i \neq j}\left(k_{i} \epsilon_{q}\right)\left(k_{j} \bar{\epsilon}_{q}\right) \sum_{l \neq i}\left(k_{l} q\right) \mathcal{G}\left(z_{l}, z_{j}\right)\left[\mathcal{G}\left(z_{l}, z_{i}\right)-\mathcal{G}\left(z_{j}, z_{i}\right)\right]
\end{aligned}
$$

This removes, as promised, the divergent terms for $l=j$. In the last line $l=j$ is evidently zero, and the sum over $l$ can be reduces to $l \neq i, j$.

By summing the (blue) first term in the last line of eq. (E.19) with the second-to-last line in eq. (E.17) (also blue), we get:

$$
\begin{aligned}
& -\sum_{i}\left(k_{i} \epsilon_{q}\right)\left(k_{i} \bar{\epsilon}_{q}\right) \int d^{2} z \mathcal{T}(z) \mathcal{G}\left(z, z_{i}\right)-\sum_{i \neq j}\left(k_{i} \epsilon_{q}\right)\left(k_{j} \bar{\epsilon}_{q}\right) \int d^{2} z \mathcal{T}(z) \mathcal{G}\left(z, z_{j}\right) \\
& =-\sum_{i, j}\left(k_{i} \epsilon_{q}\right)\left(k_{j} \bar{\epsilon}_{q}\right) \int d^{2} z \mathcal{T}(z) \mathcal{G}\left(z, z_{j}\right)=\sum_{j}\left(q \epsilon_{q}\right)\left(k_{j} \bar{\epsilon}_{q}\right) \int d^{2} z \mathcal{T}(z) \mathcal{G}\left(z, z_{j}\right)=0
\end{aligned}
$$

By collecting the results for all $i$ and $j$ we get:

$$
\begin{aligned}
I_{1}= & 2 \pi \sum_{i} \frac{\left(k_{i} \epsilon_{q}\right)\left(k_{i} \bar{\epsilon}_{q}\right)}{k_{i} q} \sum_{l \neq i}\left(k_{l} q\right) \mathcal{G}\left(z_{l}, z_{i}\right)+\frac{2 \pi \alpha^{\prime}}{4} \sum_{i}\left(k_{i} \epsilon_{q}\right)\left(k_{i} \bar{\epsilon}_{q}\right) \sum_{l \neq i}\left(k_{l} q\right) \mathcal{G}^{2}\left(z_{l}, z_{i}\right) \\
& +\frac{2 \pi \alpha^{\prime}}{2} \sum_{i \neq j}\left(k_{i} \epsilon_{q}\right)\left(k_{j} \bar{\epsilon}_{q}\right) \sum_{l \neq i}\left(k_{l} q\right) \mathcal{G}\left(z_{l}, z_{j}\right)\left[\mathcal{G}\left(z_{l}, z_{i}\right)-\mathcal{G}\left(z_{j}, z_{i}\right)\right] \\
& -\frac{\alpha^{\prime}}{2} \sum_{i \neq j}\left(k_{i} \epsilon_{q}\right)\left(k_{j} \bar{\epsilon}_{q}\right) \sum_{l \neq i}\left(k_{l} q\right) \int d^{2} z \partial_{\bar{z}}\left[\partial_{z} \mathcal{G}\left(z, z_{l}\right) \mathcal{G}\left(z, z_{j}\right)\right] \mathcal{G}\left(z, z_{i}\right) \\
& -\frac{\alpha^{\prime}}{4} \sum_{i}\left(k_{i} \epsilon_{q}\right)\left(k_{i} \bar{\epsilon}_{q}\right)\left(k_{i} q\right) \int d^{2} z \mathcal{T}(z) \mathcal{G}^{2}\left(z, z_{i}\right) \\
& -\frac{\alpha^{\prime}}{2} \sum_{i \neq j}\left(k_{i} \epsilon_{q}\right)\left(k_{j} \bar{\epsilon}_{q}\right) k_{i} q \int d^{2} z \mathcal{T}(z) \mathcal{G}\left(z, z_{j}\right) \mathcal{G}\left(z, z_{i}\right)+O\left(q^{2}\right)
\end{aligned}
$$

The first term is the only one of order $q^{0}$ and it is formally identical to the tree-level results. There are still three integrals to be computed at the order $q$. We will show that the first of 
these, not involving $\mathcal{T}$ explicitly, can be computed in the case of a symmetrically polarized soft external states up to terms that will cancel agains the remaining two integrals involving $\mathcal{T}$ explicitly.

First we split the integral in two parts, for $l \neq j$ and $l=j$ :

$$
\begin{aligned}
I_{1,1}= & -\frac{\alpha^{\prime}}{2} \sum_{i \neq j}\left(k_{i} \epsilon_{q}\right)\left(k_{j} \bar{\epsilon}_{q}\right) \sum_{l \neq i, j}\left(k_{l} q\right) \int d^{2} z \partial_{\bar{z}}\left[\partial_{z} \mathcal{G}\left(z, z_{l}\right) \mathcal{G}\left(z, z_{j}\right)\right] \mathcal{G}\left(z, z_{i}\right) \\
& -\frac{\alpha^{\prime}}{2} \sum_{i \neq j}\left(k_{i} \epsilon_{q}\right)\left(k_{j} \bar{\epsilon}_{q}\right)\left(k_{j} q\right) \int d^{2} z \partial_{\bar{z}}\left[\partial_{z} \mathcal{G}\left(z, z_{j}\right) \mathcal{G}\left(z, z_{j}\right)\right] \mathcal{G}\left(z, z_{i}\right)
\end{aligned}
$$

The case $l=j$ can be rewritten as:

$$
\begin{aligned}
\left.I_{1,1}\right|_{l=j} & =-\frac{\alpha^{\prime}}{4} \sum_{i \neq j}\left(k_{i} \epsilon_{q}\right)\left(k_{j} \bar{\epsilon}_{q}\right)\left(k_{j} q\right) \int d^{2} z \partial_{\bar{z}} \partial_{z} \mathcal{G}^{2}\left(z, z_{j}\right) \mathcal{G}\left(z, z_{i}\right) \\
& =+\frac{\alpha^{\prime}}{4} \sum_{i \neq j}\left(k_{i} \epsilon_{q}\right)\left(k_{j} \bar{\epsilon}_{q}\right)\left(k_{j} q\right) \int d^{2} z \partial_{z} \mathcal{G}^{2}\left(z, z_{j}\right) \partial_{\bar{z}} \mathcal{G}\left(z, z_{i}\right) \\
& =-\frac{\alpha^{\prime}}{4} \sum_{i \neq j}\left(k_{i} \epsilon_{q}\right)\left(k_{j} \bar{\epsilon}_{q}\right)\left(k_{j} q\right) \int d^{2} z \mathcal{G}^{2}\left(z, z_{j}\right) \partial_{z} \partial_{\bar{z}} \mathcal{G}\left(z, z_{i}\right) \\
& =-\frac{\alpha^{\prime}}{4} \sum_{i \neq j}\left(k_{i} \epsilon_{q}\right)\left(k_{j} \bar{\epsilon}_{q}\right)\left(k_{j} q\right)\left[2 \pi \mathcal{G}^{2}\left(z_{i}, z_{j}\right)+\int d^{2} z \mathcal{T}(z) \mathcal{G}^{2}\left(z, z_{j}\right)\right]
\end{aligned}
$$

The second case $l \neq j$ in eq. (E.23) can be computed for the soft graviton and dilaton without knowing $\mathcal{G}$ explicitly. Using the symmetry of the polarization tensor, we can write it as:

$$
\begin{aligned}
\left.I_{1,1}\right|_{l \neq j}= & -\frac{\alpha^{\prime}}{4} \sum_{i \neq j} k_{i}^{\mu} k_{j}^{\nu} \epsilon_{q \mu \nu}^{S} \sum_{l \neq i, j}\left(k_{l} q\right) \int d^{2} z \partial_{\bar{z}}\left[\partial_{z} \mathcal{G}\left(z, z_{l}\right) \mathcal{G}\left(z, z_{j}\right)\right] \mathcal{G}\left(z, z_{i}\right) \\
& -\frac{\alpha^{\prime}}{4} \sum_{i \neq j} k_{i}^{\mu} k_{j}^{\nu} \epsilon_{q \mu \nu}^{S} \sum_{l \neq i, j}\left(k_{l} q\right) \int d^{2} z \partial_{\bar{z}}\left[\partial_{z} \mathcal{G}\left(z, z_{l}\right) \mathcal{G}\left(z, z_{i}\right)\right] \mathcal{G}\left(z, z_{j}\right)
\end{aligned}
$$

and by partial integration we get:

$$
\begin{aligned}
\left.I_{1,1}\right|_{l \neq j}= & +\frac{\alpha^{\prime}}{4} \sum_{i \neq j} k_{i}^{\mu} k_{j}^{\nu} \epsilon_{q \mu \nu}^{S} \sum_{l \neq i, j}\left(k_{l} q\right) \int d^{2} z \partial_{z} \mathcal{G}\left(z, z_{l}\right) \mathcal{G}\left(z, z_{j}\right) \partial_{\bar{z}} \mathcal{G}\left(z, z_{i}\right) \\
& +\frac{\alpha^{\prime}}{4} \sum_{i \neq j} k_{i}^{\mu} k_{j}^{\nu} \epsilon_{q \mu \nu}^{S} \sum_{l \neq i, j}\left(k_{l} q\right) \int d^{2} z \partial_{z} \mathcal{G}\left(z, z_{l}\right) \mathcal{G}\left(z, z_{i}\right) \partial_{\bar{z}} \mathcal{G}\left(z, z_{j}\right) \\
= & +\frac{\alpha^{\prime}}{4} \sum_{i \neq j} k_{i}^{\mu} k_{j}^{\nu} \epsilon_{q \mu \nu}^{S} \sum_{l \neq i, j}\left(k_{l} q\right) \int d^{2} z \partial_{z} \mathcal{G}\left(z, z_{l}\right) \partial_{\bar{z}}\left[\mathcal{G}\left(z, z_{i}\right) \mathcal{G}\left(z, z_{j}\right)\right] \\
= & -\frac{\alpha^{\prime}}{4} \sum_{i \neq j} k_{i}^{\mu} k_{j}^{\nu} \epsilon_{q \mu \nu}^{S} \sum_{l \neq i, j}\left(k_{l} q\right) \int d^{2} z \partial_{\bar{z}} \partial_{z} \mathcal{G}\left(z, z_{l}\right) \mathcal{G}\left(z, z_{j}\right) \mathcal{G}\left(z, z_{i}\right)
\end{aligned}
$$


Finally, we can make use of eq. (E.1) and get

$$
\begin{aligned}
\left.I_{1,1}\right|_{l \neq j}= & -\frac{2 \pi \alpha^{\prime}}{4} \sum_{i \neq j} k_{i}^{\mu} k_{j}^{\nu} \epsilon_{q \mu \nu}^{S} \sum_{l \neq i, j}\left(k_{l} q\right) \mathcal{G}\left(z_{l}, z_{j}\right) \mathcal{G}\left(z_{l}, z_{i}\right) \\
& -\frac{\alpha^{\prime}}{4} \sum_{i \neq j} k_{i}^{\mu} k_{j}^{\nu} \epsilon_{q \mu \nu}^{S}\left(-k_{i} q-k_{j} q\right) \int d^{2} z \mathcal{T}(z) \mathcal{G}\left(z, z_{j}\right) \mathcal{G}\left(z, z_{i}\right)
\end{aligned}
$$

In the last line we used $\sum_{l \neq i, j} k_{l} q=-\left(k_{i} q+k_{j} q\right)$. Summing this expression with the contribution from eq. (E.24) for a symmetrically polarized soft state we get:

$$
\begin{aligned}
I_{1,1}=- & \frac{\alpha^{\prime}}{4} \epsilon_{q \mu \nu}^{S} \sum_{i \neq j} k_{i}^{\mu} k_{j}^{\nu}\left[2 \pi \sum_{l \neq i, j}\left(k_{l} q\right) \mathcal{G}\left(z_{l}, z_{j}\right) \mathcal{G}\left(z_{l}, z_{i}\right)+2 \pi\left(k_{j} q\right) \mathcal{G}^{2}\left(z_{i}, z_{j}\right)\right. \\
& \left.-2\left(k_{i} q\right) \int d^{2} z \mathcal{T}(z) \mathcal{G}\left(z, z_{j}\right) \mathcal{G}\left(z, z_{i}\right)\right]+\frac{\alpha^{\prime}}{4} \epsilon_{q \mu \nu}^{S} \sum_{j=1} k_{j}^{\mu} k_{j}^{\nu}\left(k_{j} q\right) \int d^{2} z \mathcal{T}(z) \mathcal{G}^{2}\left(z, z_{j}\right)
\end{aligned}
$$

where in the last term we used $\sum_{i \neq j}\left(k_{i} \epsilon_{q}\right)=-\left(k_{j} \epsilon_{q}\right)$. It is evident that this term exactly cancels the similar (purple) term in eq. (E.22), while the third (orange) term above for a symmetrically polarized soft state exactly cancels the similar (also orange) term in eq. (E.22). The final result for $I_{1}$ when the soft state is symmetrically polarized is thus

$$
\begin{aligned}
I_{1}=2 \pi \epsilon_{q \mu \nu}^{S} \sum_{i \neq j}[ & \frac{k_{i}^{\mu} k_{i}^{\nu}}{k_{i} q}\left(k_{j} q\right) \mathcal{G}\left(z_{j}, z_{i}\right)+\frac{\alpha^{\prime}}{4} k_{i}^{\mu} k_{i}^{\nu}\left(k_{j} q\right) \mathcal{G}^{2}\left(z_{j}, z_{i}\right) \\
& +\frac{\alpha^{\prime}}{2} k_{i}^{\mu} k_{j}^{\nu} \sum_{l \neq i, j}\left(k_{l} q\right) \mathcal{G}\left(z_{l}, z_{j}\right)\left(\mathcal{G}\left(z_{l}, z_{i}\right)-\mathcal{G}\left(z_{j}, z_{i}\right)\right) \\
& \left.-\frac{\alpha^{\prime}}{4} k_{i}^{\mu} k_{j}^{\nu} \sum_{l \neq i, j}\left(k_{l} q\right) \mathcal{G}\left(z_{l}, z_{j}\right) \mathcal{G}\left(z_{l}, z_{i}\right)-\frac{\alpha^{\prime}}{4} k_{i}^{\mu} k_{j}^{\nu}\left(k_{j} q\right) \mathcal{G}^{2}\left(z_{i}, z_{j}\right)\right]
\end{aligned}
$$

It is useful to rewrite the terms with sum over the label $l$ as follows:

$$
\begin{gathered}
\frac{2 \pi \alpha^{\prime}}{2} \epsilon_{q \mu \nu}^{S} \sum_{i \neq j \neq l} k_{i}^{\mu} k_{j}^{\nu}\left(k_{l} q\right)\left[\mathcal{G}\left(z_{l}, z_{j}\right)\left(\mathcal{G}\left(z_{l}, z_{i}\right)-\mathcal{G}\left(z_{j}, z_{i}\right)\right)-\frac{1}{2} \mathcal{G}\left(z_{l}, z_{j}\right) \mathcal{G}\left(z_{l}, z_{i}\right)\right] \\
=\frac{2 \pi \alpha^{\prime}}{4} \epsilon_{q \mu \nu}^{S} \sum_{i \neq j \neq l}\left[k_{j}^{\mu} k_{l}^{\nu}\left(k_{i} q\right)-k_{i}^{\mu} k_{l}^{\nu}\left(k_{j} q\right)-k_{i}^{\mu} k_{j}^{\nu}\left(k_{l} q\right)\right] \mathcal{G}\left(z_{i}, z_{l}\right) \mathcal{G}\left(z_{i}, z_{j}\right)
\end{gathered}
$$

The first term can be joined with the second term in eq. (E.29) by extending the sum to include $l=j$, while the third term can be joined with the last term in eq. (E.29) by extending it to include $l=j$. Thus we can write:

$$
\begin{aligned}
I_{1}= & 2 \pi \epsilon_{q \mu \nu}^{S} \sum_{i \neq j} \frac{k_{i}^{\mu} k_{i}^{\nu}}{k_{i} q}\left(k_{j} q\right) \mathcal{G}\left(z_{j}, z_{i}\right)+\frac{2 \pi \alpha^{\prime}}{4} \epsilon_{q \mu \nu}^{S} \sum_{i \neq j} k_{i}^{\mu} k_{j}^{\nu}\left(k_{j} q\right) \mathcal{G}^{2}\left(z_{i}, z_{j}\right) \\
& +\frac{2 \pi \alpha^{\prime}}{4} \epsilon_{q \mu \nu}^{S} \sum_{i \neq j, l}\left[k_{j}^{\mu} k_{l}^{\nu}\left(k_{i} q\right)-k_{i}^{\mu} k_{l}^{\nu}\left(k_{j} q\right)-k_{i}^{\mu} k_{j}^{\nu}\left(k_{l} q\right)\right] \mathcal{G}\left(z_{i}, z_{l}\right) \mathcal{G}\left(z_{i}, z_{j}\right)
\end{aligned}
$$

The result of this integral is exactly the same as the tree-level result with the difference all being in the Green function. In particular, all dependence on $\mathcal{T}(z)$ have either cancelled or vanished. 


\section{E.3 Calculation of $I_{2}$}

The last integral to evaluate in eq. (3.3) is:

$$
I_{2}=\frac{\alpha^{\prime}}{2} \sum_{i, j}\left(k_{i} \epsilon_{q}\right)\left(k_{j} \bar{\epsilon}_{q}\right) \sum_{l, k \neq i}\left(k_{l} q\right)\left(k_{k} q\right) \int d^{2} z \mathcal{G}\left(z, z_{l}\right) \mathcal{G}\left(z, z_{k}\right) \partial_{z} \mathcal{G}\left(z, z_{i}\right) \partial_{\bar{z}} \mathcal{G}\left(z, z_{j}\right) e^{\frac{\alpha^{\prime}}{2} k_{i} q \mathcal{G}\left(z, z_{i}\right)}
$$

Since it has two factors of $q$ in front of the integral, we only need to extract the leading $1 / q$ behavior of the integral. Considering only the integral, denoted by $\mathcal{I}_{i j k l}$, let us first consider the case when $j \neq i$. In that case we can write the integrand as

$$
\begin{aligned}
\left.\mathcal{I}_{i j k l}\right|_{j \neq i} & =\frac{\alpha^{\prime}}{2} \int d^{2} z \mathcal{G}\left(z, z_{l}\right) \mathcal{G}\left(z, z_{k}\right) \partial_{z} \mathcal{G}\left(z, z_{i}\right) \partial_{\bar{z}} \mathcal{G}\left(z, z_{j}\right) e^{\frac{\alpha^{\prime}}{2} k_{i} q \mathcal{G}\left(z, z_{i}\right)} \\
& =\frac{1}{k_{i} q} \int d^{2} z \mathcal{G}\left(z, z_{l}\right) \mathcal{G}\left(z, z_{k}\right) \partial_{\bar{z}} \mathcal{G}\left(z, z_{j}\right) \partial_{z} e^{\frac{\alpha^{\prime}}{2} k_{i} q \mathcal{G}\left(z, z_{i}\right)} \\
& =-\frac{1}{k_{i} q} \int d^{2} z \partial_{z}\left[\mathcal{G}\left(z, z_{l}\right) \mathcal{G}\left(z, z_{k}\right) \partial_{\bar{z}} \mathcal{G}\left(z, z_{j}\right)\right] e^{\frac{\alpha^{\prime}}{2} k_{i} q \mathcal{G}\left(z, z_{i}\right)} \\
& =0+\mathcal{O}\left(q^{0}\right)
\end{aligned}
$$

where in the last equality we expanded the exponential (since in the bracket there is no singularity at $z=z_{i}$ ), and the leading term vanishes because it is a total derivative.

To compute the case $i=j$ observe that

$$
\begin{aligned}
& \partial_{z} \partial_{\bar{z}} e^{\frac{\alpha^{\prime}}{2} q k_{i} \mathcal{G}\left(z, z_{i}\right)}=\partial_{z}\left[\frac{\alpha^{\prime}}{2} k_{i} q \partial_{\bar{z}} \mathcal{G}\left(z, z_{i}\right) e^{\frac{\alpha^{\prime}}{2} q k_{i} \mathcal{G}\left(z, z_{i}\right)}\right] \\
& =\frac{\alpha^{\prime}}{2} k_{i} q \partial_{z} \partial_{\bar{z}} \mathcal{G}\left(z, z_{i}\right) e^{\frac{\alpha^{\prime}}{2} k_{i} q \mathcal{G}\left(z, z_{i}\right)}+\left(\frac{\alpha^{\prime}}{2} k_{i} q\right)^{2} \partial_{\bar{z}} \mathcal{G}\left(z, z_{i}\right) \partial_{z} \mathcal{G}\left(z, z_{i}\right) e^{\frac{\alpha^{\prime}}{2} k_{i} q \mathcal{G}\left(z, z_{i}\right)}
\end{aligned}
$$

Thus we can make the following rewriting of the integral:

$$
\begin{aligned}
\left.\mathcal{I}_{i j k l}\right|_{j=i}= & \frac{1}{\frac{\alpha^{\prime}}{2}\left(q k_{i}\right)^{2}} \int d^{2} z \mathcal{G}\left(z, z_{l}\right) \mathcal{G}\left(z, z_{k}\right) \partial_{z} \partial_{\bar{z}} e^{\frac{\alpha^{\prime}}{2} q k_{i} \mathcal{G}\left(z, z_{i}\right)} \\
& -\frac{1}{q k_{i}} \int d^{2} z \mathcal{G}\left(z, z_{l}\right) \mathcal{G}\left(z, z_{k}\right) \partial_{z} \partial_{\bar{z}} \mathcal{G}\left(z, z_{i}\right) e^{\frac{\alpha^{\prime}}{2} q k_{i} \mathcal{G}\left(z, z_{i}\right)} \\
= & -\frac{1}{\frac{\alpha^{\prime}}{2}\left(q k_{i}\right)^{2}} \int d^{2} z \partial_{z}\left[\mathcal{G}\left(z, z_{l}\right) \mathcal{G}\left(z, z_{k}\right)\right] \partial_{\bar{z}} e^{\frac{\alpha^{\prime}}{2} q k_{i} \mathcal{G}\left(z, z_{i}\right)} \\
& -\frac{1}{q k_{i}} \int d^{2} z \mathcal{T}(z) \mathcal{G}\left(z, z_{l}\right) \mathcal{G}\left(z, z_{k}\right) e^{\frac{\alpha^{\prime}}{2} q k_{i} \mathcal{G}\left(z, z_{i}\right)}
\end{aligned}
$$

where total derivatives were set to zero and we made use of the identity (E.3). We can now expand the exponentials, since $i \neq l, k$, and the leading order contributions read:

$$
\begin{aligned}
\left.\mathcal{I}_{i j k l}\right|_{j=i}= & \frac{1}{\frac{\alpha^{\prime}}{2} q k_{i}} \int d^{2} z \partial_{\bar{z}} \partial_{z}\left(\mathcal{G}\left(z, z_{l}\right) \mathcal{G}\left(z, z_{k}\right)\right) \mathcal{G}\left(z, z_{i}\right) \\
& -\frac{1}{\frac{\alpha^{\prime}}{2} q k_{i}} \int d^{2} z \mathcal{T}(z) \mathcal{G}\left(z, z_{l}\right) \mathcal{G}\left(z, z_{k}\right)+O\left(q^{0}\right)
\end{aligned}
$$


A double integration by parts of the first terms gives:

$$
\begin{aligned}
\left.\mathcal{I}_{i j k l}\right|_{j=i}= & \frac{2 \pi}{\frac{\alpha^{\prime}}{2} q k_{i}} \mathcal{G}\left(z_{i}, z_{l}\right) \mathcal{G}\left(z_{i}, z_{k}\right)+\frac{1}{\frac{\alpha^{\prime}}{2} q k_{i}} \int d^{2} z \mathcal{T}(z) \mathcal{G}\left(z, z_{l}\right) \mathcal{G}\left(z, z_{k}\right) \\
& -\frac{1}{\frac{\alpha^{\prime}}{2} q k_{i}} \int d^{2} z \mathcal{T}(z) \mathcal{G}\left(z, z_{l}\right) \mathcal{G}\left(z, z_{k}\right)+O\left(q^{0}\right)
\end{aligned}
$$

Hence the two terms involving $\mathcal{T}$ cancel.

We have thus extracted all $1 / q$ dependence of the integral and the final result for $I_{2}$ reads:

$$
I_{2}=2 \pi \sum_{i=1}\left(k_{i} \epsilon_{q}\right)\left(k_{i} \bar{\epsilon}_{q}\right) \sum_{l, k \neq i} \frac{\left(k_{l} q\right)\left(k_{k} q\right)}{q k_{i}} \mathcal{G}\left(z_{i}, z_{l}\right) \mathcal{G}\left(z_{i}, z_{k}\right)
$$

This result is formally identical with the tree-level result with all the difference being in the Green function.

\section{F Calculation of $S_{2}^{(1)}$}

In this appendix we consider, at one loop, the integral that appears in eq. (3.7). For the calculation we have made explicit use of the one loop expression, and we have not been able to extend this to the generic multiloop level.

The integral in eq. (3.7) can be easily evaluated by performing the change of variable $z=e^{2 \pi i \nu}$, and by using the one-loop expression of Green's function given for example in eq. (3.8) of ref. [87]. The result of the calculation is an expression depending only on the moduli of the torus that makes eq. (3.7) zero on-shell, since $\sum_{l=1}^{N} k_{l} q=-q^{2}=0$. In the following instead of giving the details of the calculation, we will prove the independence of the integral on the variable $z_{l}$, i.e.:

$$
\partial_{z_{l}} \int_{\mathcal{A}} d^{2} z \omega(z) \bar{\omega}(\bar{z}) \mathcal{G}_{1}\left(z, z_{l}\right)=0
$$

This is sufficient to show the vanishing of eq. (3.7) on shell. Here $\mathcal{A}=\{z \in \mathbb{C}$, s.t. $|\kappa| \leq$ $|z| \leq 1\}$ denotes the one-loop integration region. In order to prove eq. (F.1), we first observe that, with the choice of $V_{i}^{\prime}(0)=2 \pi z_{i}$, the Green function satisfies the identity [88]:

$$
\omega\left(z_{l}\right) \partial_{z} \mathcal{G}_{1}\left(z, z_{l}\right)+\omega(z) \partial_{z_{l}} \mathcal{G}_{1}\left(z, z_{l}\right)=0
$$

that, when used in eq. (F.1), gives:

$$
-\int_{\mathcal{A}} d^{2} z \partial_{z} \frac{\mathcal{G}_{1}\left(z, z_{l}\right)}{\bar{z} z_{l}}=-\frac{1}{2 i z_{l}}\left[\oint_{|z|=1} \frac{d \bar{z}}{\bar{z}} \mathcal{G}_{1}\left(z, z_{l}\right)-\oint_{|z|=|\kappa|} \frac{d \bar{z}}{\bar{z}} \mathcal{G}_{1}\left(z, z_{l}\right)\right]
$$

In the right-hand-side of the previous expression one can change the variable $\bar{z}=\bar{z}^{\prime} \bar{\kappa}$, getting:

$$
-\int_{\mathcal{A}} d^{2} z \partial_{z} \frac{\mathcal{G}_{1}\left(z, z_{l}\right)}{\bar{z} z_{l}}=-\frac{1}{2 i z_{l}} \oint_{|z|=1} \frac{d \bar{z}}{\bar{z}}\left[\mathcal{G}_{1}\left(z, z_{l}\right)-\mathcal{G}_{1}\left(k z, z_{l}\right)\right]=0
$$

The expression is zero from the invariance of the Green function under transport around the $b$-cycle of the torus, i.e. $\mathcal{G}_{1}\left(z, z_{l}\right)=\mathcal{G}_{1}\left(k z, z_{l}\right)$. 
The multiloop extension of such a proof would require the multiloop generalization of eq. (F.1). At one loop this follows from the identity (F.2) and from the invariance of the Green function along the homology cycles of the Riemann surface. The main obstacle to extend these considerations to arbitrary orders in the perturbative expansion, is the lack of a multiloop identity similar to eq. (F.2).

Open Access. This article is distributed under the terms of the Creative Commons Attribution License (CC-BY 4.0), which permits any use, distribution and reproduction in any medium, provided the original author(s) and source are credited.

\section{References}

[1] A. Strominger, Asymptotic symmetries of Yang-Mills theory, JHEP 07 (2014) 151 [arXiv:1308.0589] [INSPIRE].

[2] A. Strominger, On BMS invariance of gravitational scattering, JHEP 07 (2014) 152 [arXiv: 1312.2229] [INSPIRE].

[3] T. He, V. Lysov, P. Mitra and A. Strominger, BMS supertranslations and Weinberg's soft graviton theorem, JHEP 05 (2015) 151 [arXiv: 1401.7026] [INSPIRE].

[4] F. Cachazo and A. Strominger, Evidence for a new soft graviton theorem, arXiv:1404.4091 [INSPIRE].

[5] B.U.W. Schwab and A. Volovich, Subleading soft theorem in arbitrary dimensions from scattering equations, Phys. Rev. Lett. 113 (2014) 101601 [arXiv:1404.7749] [inSPIRE].

[6] M. Zlotnikov, Sub-sub-leading soft-graviton theorem in arbitrary dimension, JHEP 10 (2014) 148 [arXiv:1407.5936] [INSPIRE].

[7] C. Kalousios and F. Rojas, Next to subleading soft-graviton theorem in arbitrary dimensions, JHEP 01 (2015) 107 [arXiv:1407.5982] [INSPIRE].

[8] M. Campiglia and A. Laddha, Asymptotic symmetries and subleading soft graviton theorem, Phys. Rev. D 90 (2014) 124028 [arXiv:1408.2228] [inSPIRE].

[9] Y.-J. Du, B. Feng, C.-H. Fu and Y. Wang, Note on soft graviton theorem by KLT relation, JHEP 11 (2014) 090 [arXiv: 1408.4179] [INSPIRE].

[10] M. Campiglia and A. Laddha, Asymptotic symmetries of gravity and soft theorems for massive particles, JHEP 12 (2015) 094 [arXiv: 1509. 01406] [INSPIRE].

[11] A. Strominger and A. Zhiboedov, Gravitational memory, BMS supertranslations and soft theorems, JHEP 01 (2016) 086 [arXiv:1411.5745] [INSPIRE].

[12] M. Campiglia and A. Laddha, New symmetries for the gravitational S-matrix, JHEP 04 (2015) 076 [arXiv:1502.02318] [INSPIRE].

[13] D. Kapec, V. Lysov, S. Pasterski and A. Strominger, Higher-dimensional supertranslations and Weinberg's soft graviton theorem, AMSA 2 (2017) 69 [arXiv:1502.07644] [INSPIRE].

[14] T. Klose, T. McLoughlin, D. Nandan, J. Plefka and G. Travaglini, Double-soft limits of gluons and gravitons, JHEP 07 (2015) 135 [arXiv: 1504.05558] [INSPIRE].

[15] M. Campiglia and A. Laddha, Asymptotic symmetries of QED and Weinberg's soft photon theorem, JHEP 07 (2015) 115 [arXiv: 1505.05346] [INSPIRE]. 
[16] M. Campiglia and A. Laddha, Sub-subleading soft gravitons: new symmetries of quantum gravity?, Phys. Lett. B 764 (2017) 218 [arXiv:1605.09094] [INSPIRE].

[17] M. Campiglia and A. Laddha, Subleading soft photons and large gauge transformations, JHEP 11 (2016) 012 [arXiv: 1605.09677] [INSPIRE].

[18] M. Campiglia and A. Laddha, Sub-subleading soft gravitons and large diffeomorphisms, JHEP 01 (2017) 036 [arXiv:1608.00685] [INSPIRE].

[19] A. Luna, S. Melville, S.G. Naculich and C.D. White, Next-to-soft corrections to high energy scattering in QCD and gravity, JHEP 01 (2017) 052 [arXiv:1611.02172] [INSPIRE].

[20] H. Elvang, C.R.T. Jones and S.G. Naculich, Soft photon and graviton theorems in effective field theory, Phys. Rev. Lett. 118 (2017) 231601 [arXiv:1611.07534] [INSPIRE].

[21] E. Conde and P. Mao, BMS supertranslations and not so soft gravitons, JHEP 05 (2017) 060 [arXiv: 1612.08294] [INSPIRE].

[22] M. Campiglia, L. Coito and S. Mizera, Can scalars have asymptotic symmetries?, Phys. Rev. D 97 (2018) 046002 [arXiv:1703.07885] [INSPIRE].

[23] P. Di Vecchia, R. Marotta and M. Mojaza, Double-soft behavior of the dilaton of spontaneously broken conformal invariance, JHEP 09 (2017) 001 [arXiv:1705.06175] [INSPIRE].

[24] A.L. Guerrieri, Y.-T. Huang, Z. Li and C. Wen, On the exactness of soft theorems, JHEP 12 (2017) 052 [arXiv: 1705.10078] [INSPIRE].

[25] S. Chakrabarti, S.P. Kashyap, B. Sahoo, A. Sen and M. Verma, Subleading soft theorem for multiple soft gravitons, JHEP 12 (2017) 150 [arXiv:1707.06803] [INSPIRE].

[26] Y. Hamada and S. Sugishita, Soft pion theorem, asymptotic symmetry and new memory effect, JHEP 11 (2017) 203 [arXiv:1709.05018] [INSPIRE].

[27] S. Chakrabarti, S.P. Kashyap, B. Sahoo, A. Sen and M. Verma, Testing subleading multiple soft graviton theorem for CHY prescription, JHEP 01 (2018) 090 [arXiv:1709. 07883] [INSPIRE].

[28] Z.-Z. Li, H.-H. Lin and S.-Q. Zhang, On the symmetry foundation of double soft theorems, JHEP 12 (2017) 032 [arXiv:1710.00480] [INSPIRE].

[29] Y. Hamada and G. Shiu, Infinite set of soft theorems in gauge-gravity theories as Ward-Takahashi identities, Phys. Rev. Lett. 120 (2018) 201601 [arXiv:1801.05528] [INSPIRE].

[30] F. Loebbert, M. Mojaza and J. Plefka, Hidden conformal symmetry in tree-level graviton scattering, JHEP 05 (2018) 208 [arXiv: 1802.05999] [INSPIRE].

[31] A.H. Anupam, A. Kundu and K. Ray, Double soft graviton theorems and Bondi-Metzner-Sachs symmetries, Phys. Rev. D 97 (2018) 106019 [arXiv:1803.03023] [INSPIRE].

[32] S. Banerjee, Symmetries of free massless particles and soft theorems, arXiv:1804.06646 [INSPIRE].

[33] L. Rodina, Scattering amplitudes from soft theorems and infrared behavior, arXiv: 1807.09738 [INSPIRE].

[34] B. Sahoo and A. Sen, Classical and quantum results on logarithmic terms in the soft theorem in four dimensions, arXiv: 1808.03288 [INSPIRE]. 
[35] M. Bianchi, S. He, Y.-T. Huang and C. Wen, More on soft theorems: trees, loops and strings, Phys. Rev. D 92 (2015) 065022 [arXiv: 1406.5155] [inSPIRE].

[36] B.U.W. Schwab, A note on soft factors for closed string scattering, JHEP 03 (2015) 140 [arXiv: 1411.6661] [INSPIRE].

[37] M. Bianchi and A.L. Guerrieri, On the soft limit of open string disk amplitudes with massive states, JHEP 09 (2015) 164 [arXiv:1505.05854] [INSPIRE].

[38] S.G. Avery and B.U.W. Schwab, Burg-Metzner-Sachs symmetry, string theory and soft theorems, Phys. Rev. D 93 (2016) 026003 [arXiv: 1506. 05789] [inSPIRE].

[39] P. Di Vecchia, R. Marotta and M. Mojaza, Double-soft behavior for scalars and gluons from string theory, JHEP 12 (2015) 150 [arXiv:1507.00938] [INSPIRE].

[40] A.L. Guerrieri, Soft behavior of string amplitudes with external massive states, in $27^{\text {th }}$ Conference on High Energy Physics (IFAE 2015), Rome, Italy, 8-10 April 2015 [Nuovo Cim. C 39 (2016) 221] [arXiv:1507.08829] [INSPIRE].

[41] P. Di Vecchia, R. Marotta and M. Mojaza, Soft theorems from string theory, Fortsch. Phys. 64 (2016) 389 [arXiv:1511.04921] [INSPIRE].

[42] M. Bianchi and A.L. Guerrieri, On the soft limit of closed string amplitudes with massive states, Nucl. Phys. B 905 (2016) 188 [arXiv:1512.00803] [INSPIRE].

[43] A. Sen, Soft theorems in superstring theory, JHEP 06 (2017) 113 [arXiv: 1702.03934] [INSPIRE].

[44] S. Higuchi and H. Kawai, Universality of soft theorem from locality of soft vertex operators, Nucl. Phys. B 936 (2018) 400 [arXiv: 1805.11079] [INSPIRE].

[45] P. Di Vecchia, R. Marotta, M. Mojaza and J. Nohle, New soft theorems for the gravity dilaton and the Nambu-Goldstone dilaton at subsubleading order, Phys. Rev. D 93 (2016) 085015 [arXiv:1512.03316] [INSPIRE].

[46] P. Di Vecchia, R. Marotta and M. Mojaza, Soft behavior of a closed massless state in superstring and universality in the soft behavior of the dilaton, JHEP 12 (2016) 020 [arXiv: 1610.03481] [INSPIRE].

[47] J. Broedel, M. de Leeuw, J. Plefka and M. Rosso, Constraining subleading soft gluon and graviton theorems, Phys. Rev. D 90 (2014) 065024 [arXiv:1406.6574] [INSPIRE].

[48] Z. Bern, S. Davies, P. Di Vecchia and J. Nohle, Low-energy behavior of gluons and gravitons from gauge invariance, Phys. Rev. D 90 (2014) 084035 [arXiv:1406.6987] [InSPIRE].

[49] Z.-Z. Li, H.-H. Lin and S.-Q. Zhang, Infinite soft theorems from gauge symmetry, Phys. Rev. D 98 (2018) 045004 [arXiv: 1802.03148] [INSPIRE].

[50] P. Di Vecchia, R. Marotta and M. Mojaza, Subsubleading soft theorems of gravitons and dilatons in the bosonic string, JHEP 06 (2016) 054 [arXiv: 1604.03355] [INSPIRE].

[51] P. Di Vecchia, R. Marotta and M. Mojaza, The B-field soft theorem and its unification with the graviton and dilaton, JHEP 10 (2017) 017 [arXiv:1706.02961] [INSPIRE].

[52] Z. Bern, S. Davies and J. Nohle, On loop corrections to subleading soft behavior of gluons and gravitons, Phys. Rev. D 90 (2014) 085015 [arXiv: 1405.1015] [INSPIRE].

[53] S. He, Y.-T. Huang and C. Wen, Loop corrections to soft theorems in gauge theories and gravity, JHEP 12 (2014) 115 [arXiv:1405.1410] [INSPIRE]. 
[54] J. Broedel, M. de Leeuw, J. Plefka and M. Rosso, Local contributions to factorized soft graviton theorems at loop level, Phys. Lett. B 746 (2015) 293 [arXiv:1411.2230] [InSPIRE].

[55] A. Sen, Subleading soft graviton theorem for loop amplitudes, JHEP 11 (2017) 123 [arXiv: 1703.00024] [INSPIRE].

[56] A. Laddha and A. Sen, Sub-subleading soft graviton theorem in generic theories of quantum gravity, JHEP 10 (2017) 065 [arXiv:1706.00759] [INSPIRE].

[57] P. Di Vecchia, M. Frau, A. Lerda and S. Sciuto, $N$ string vertex and loop calculation in the bosonic string, Nucl. Phys. B 298 (1988) 527 [INSPIRE].

[58] P. Di Vecchia, K. Hornfeck, M. Frau, A. Lerda and S. Sciuto, $N$ string, g loop vertex for the bosonic string, Phys. Lett. B 206 (1988) 643 [INSPIRE].

[59] P. Di Vecchia, F. Pezzella, M. Frau, K. Hornfeck, A. Lerda and A. Sciuto, $N$ point g loop vertex for a free bosonic theory with vacuum charge Q, Nucl. Phys. B 322 (1989) 317 [INSPIRE].

[60] C. Lovelace, Simple N-reggeon vertex, Phys. Lett. B 32 (1970) 490 [inSPIRE].

[61] P. Di Vecchia, R. Nakayama, J.L. Petersen, J. Sidenius and S. Sciuto, BRST invariant $N$-reggeon vertex, Phys. Lett. B 182 (1986) 164 [INSPIRE].

[62] P. Di Vecchia, M. Frau, A. Lerda and S. Sciuto, A simple expression for the multiloop amplitude in the bosonic string, Phys. Lett. B 199 (1987) 49 [INSPIRE].

[63] J.L. Petersen and J.R. Sidenius, Covariant loop calculus for the closed bosonic string, Nucl. Phys. B 301 (1988) 247 [InSPIRE].

[64] S. Mandelstam, The interacting string picture and functional integration, in Proceedings, Unified String Theories, Santa Barbara, CA, U.S.A. (1985), pg. 46 [InSPIRE].

[65] S. Mandelstam, The $N$ loop string amplitude: explicit formulas, finiteness and absence of ambiguities, Phys. Lett. B 277 (1992) 82 [INSPIRE].

[66] P. Di Vecchia, R. Marotta and M. Mojaza, Soft theorem for the graviton, dilaton and the Kalb-Ramond field in the bosonic string, JHEP 05 (2015) 137 [arXiv:1502.05258] [INSPIRE].

[67] A. Giveon, M. Porrati and E. Rabinovici, Target space duality in string theory, Phys. Rept. 244 (1994) 77 [hep-th/9401139] [INSPIRE].

[68] E. D'Hoker and D.H. Phong, The geometry of string perturbation theory, Rev. Mod. Phys. 60 (1988) 917 [inSPIRE].

[69] J.A. Shapiro, On the renormalization of dual models, Phys. Rev. D 11 (1975) 2937 [INSPIRE].

[70] M. Ademollo et al., Soft dilations and scale renormalization in dual theories, Nucl. Phys. B 94 (1975) 221 [inSPIRE].

[71] M.B. Green, J.H. Schwarz and L. Brink, $N=4$ Yang-Mills and $N=8$ supergravity as limits of string theories, Nucl. Phys. B 198 (1982) 474 [INSPIRE].

[72] S. Weinberg, Radiative corrections in string theories, talk given at the Meeting of division of particle and fields of the APS, preprint UTG-22-85, Eugene, OR, U.S.A., 15 August 1985 [INSPIRE]. 
[73] E. Cohen, H. Kluberg-Stern, H. Navelet and R.B. Peschanski, One loop renormalization of the Polyakov string functional, Nucl. Phys. B 347 (1990) 802 [InSPIRE].

[74] R. Pius, A. Rudra and A. Sen, Mass renormalization in string theory: special states, JHEP 07 (2014) 058 [arXiv: 1311.1257] [INSPIRE].

[75] R. Pius, A. Rudra and A. Sen, Mass renormalization in string theory: general states, JHEP 07 (2014) 062 [arXiv: 1401.7014] [INSPIRE].

[76] A. Sen, One loop mass renormalization of unstable particles in superstring theory, JHEP 11 (2016) 050 [arXiv:1607.06500] [INSPIRE].

[77] S. Weinberg, Infrared photons and gravitons, Phys. Rev. 140 (1965) B516 [InSPIRE].

[78] R. Akhoury, R. Saotome and G. Sterman, Collinear and soft divergences in perturbative quantum gravity, Phys. Rev. D 84 (2011) 104040 [arXiv:1109.0270] [INSPIRE].

[79] A. Laddha and A. Sen, Logarithmic terms in the soft expansion in four dimensions, JHEP 10 (2018) 056 [arXiv: 1804.09193] [INSPIRE].

[80] J. Polchinski, String theory. Volume 1: an introduction to the bosonic string, Cambridge University Press, Cambridge, U.K. (1998) [rnSPIRE].

[81] J. Polchinski, String theory. Volume 2: superstring theory and beyond, Cambridge University Press, Cambridge, U.K. (1998) [INSPIRE].

[82] T. Ortín, Gravity and strings, Cambridge University Press, Cambridge, U.K. (2015) [INSPIRE].

[83] J. Richter-Gebert, Perspectives on projective geometry, Springer, Berlin, Heidelberg, Germany (2011).

[84] L.R. Ford, Automorphic functions, MacGraw-Hill Book Company Inc., U.S.A. (1929).

[85] L. Bers, Uniformization, moduli, and Kleinian groups, Bull. London Math. Soc. 4 (1972) 257.

[86] D. Mumford, Tata lectures on theta II, Birkhäuser, Boston, MA, U.S.A. (2007).

[87] M.B. Green and P. Vanhove, The low-energy expansion of the one loop type-II superstring amplitude, Phys. Rev. D 61 (2000) 104011 [hep-th/9910056] [INSPIRE].

[88] P. Di Vecchia, L. Magnea, A. Lerda, R. Russo and R. Marotta, String techniques for the calculation of renormalization constants in field theory, Nucl. Phys. B 469 (1996) 235 [hep-th/9601143] [INSPIRE]. 